\title{
Investigation of inorganic and organic agricultural systems for Vigna spp. production in Thailand
}

\section{Duangjai Chanapan ${ }^{1}$, Sorapong Benchasri ${ }^{1 *}$ and Sakunkan Simla ${ }^{2}$}

\author{
${ }^{1}$ Department of Plant Science, Faculty of Technology and Community Development, Thaksin University, Pa \\ Phayom, Phatthalung Thailand P.O. 93210 \\ ${ }^{2}$ Department of Agricultural Technology, Faculty of Technology, Mahasarakham University, Khamriang, \\ Kantarawichai Maha Sarakham Thailand P.O. 44150
}

*Corresponding author: benchasri@gmail.com

\begin{abstract}
Yield, yield components, antioxidant activity and genetic variation of fifty genotypes on Vigna spp. were evaluated under two production systems (inorganic and organic agricultural systems) at the field trails in the Kingdom of Thailand. The experimental design was a Randomized Complete Block Design with four replications and twenty plants in blocks. It was found that pod length, fresh pod weight, number of seeds, number of pods, fresh pod yield, 100 seed weight and harvest index showed significant difference $(\mathrm{p} \leq 0.05)$. The highest quality number of pods was found on Fakdang 71.75 and 55.25 pod.plant ${ }^{-1}$ under inorganic and organic agricultural system, respectively. The Kangkragan produced the highest quality yield 1,385.20 and 1,135.30 g.plant ${ }^{-1}$ under inorganic $^{-1}$ agricultural system and organic agricultural system, respectively. Suarpran had the lowest quality yield 208.10 g.plant $^{-1}$ under organic agricultural system. The highest values for trolox equivalent antioxidant capacity (TEAC) and 2,2-diphenyl-1-picrylhydrazyl radical scavenging ability (DPPH) were obtained from Panomsarakram and Fakdang (95.36 and 35.61\% of inhibition, respectively). The outcome of this study can be used as guidance for Vigna spp. production in Thailand.
\end{abstract}

Keywords: Yield, Yield Components, Antioxidant, Genetic.

\section{Introduction}

Vigna unguiculata subsp. sesquipedalis L., yardlong bean, is a common vegetable in Asian markets (Benchasri et al., 2012), is characterized by its very long (30-90 cm in length) pods with seeds usually $8-12 \mathrm{~mm}$ long. It is also known as asparagus bean, string bean, snakebean and sitao (Kongjaimun et al., 2012). Yardlong bean originated from West Africa (Madukwe et al., 2008). It is cultivated extensively in many countries in Southeast Asia such as Malaysia, Philippines, Indonesia, Myanmar and Thailand (Win and Oo, 2015). This crop is also widely grown in Southern China, India and Pakistan. Yardlong bean could be boiled, fried or cooked (Kongjaimun et al, 2012). The composition of mature seeds per $100 \mathrm{~g}$ edible portion is: energy $47 \mathrm{Kcal}$, carbohydrates $8.35 \mathrm{~g}$, protein $2.8 \mathrm{~g}$, total fat $0.40 \mathrm{~g}$, folates $62 \mu \mathrm{g}$, niacin $0.41 \mathrm{mg}$, pantothenic acid 0.06 $\mathrm{mg}$, pyridoxine $0.02 \mathrm{mg}$, riboflavin $0.11 \mathrm{mg}$, thiamin 0.11 $\mathrm{mg}$, vitamin C $18.8 \mathrm{mg}$, vitamin A $865 \mathrm{IU}$, sodium $4 \mathrm{mg}$, potassium $240 \mathrm{mg}$, calcium50 $\mathrm{mg}$, copper $0.05 \mu \mathrm{g}$, iron 0.47 $\mathrm{mg}$, magnesium $44 \mathrm{mg}$, manganese $0.21 \mathrm{mg}$, phosphorus 59 $\mathrm{mg}$, selenium $1.5 \mu \mathrm{g}$ and zinc $0.37 \mathrm{mg}$.

Vigna unguiculata subsp. unguiculata (L.) Walp, cowpea, is classified as belonging to the same group of yardlong bean (Phansak et al., 2005), is one of the most ancient human food sources and has probably been used as a crop plant since Neolithic times (Shevkani et al., 2015). Cowpea is grown extensively in many countries (Singh et al., 2003; Win and Oo, 2015). Cowpea is a tropical grain legume which plays an important nutritional role in developing countries of the tropics and subtropics, namely sub-Saharan Africa, Asia,
Central and South America (Fang et al., 2007; Odedeji and Oyeleke, 2011; Badiane et al., 2012; Masvodza et al., 2014). Cowpea is often termed the poor man's meat because the seed protein contents range from 23 to $32 \%$ of seed weight and are rich in lysine and tryptophan, and substantial amount of mineral and vitamins, folic acid and vitamin B (Chinma et al, 2008, Huaqiang et al., 2012; Peyrano et al, 2016). The crop serves as food, animal feed, cash and manure (Henry and Chinedu, 2014). Cowpea can be regarded as a pivot of sustainable farming in regions characterized by systems of farming that make limited use of purchased inputs like inorganic fertilizer (Hamid et al, 2016). The crop can fix about $240 \mathrm{~kg} \cdot \mathrm{ha}^{-1}$ of atmospheric nitrogen and make available about $60-70 \mathrm{~kg} \cdot \mathrm{ha}^{-1}$ nitrogen for succeeding crops grown in rotation with it (Aikins and Afuakwa, 2008). Cowpea is well adapted to environmental conditions that affect crop production such as drought, high temperatures and other biotic stresses compared with other crops (Agyeman et al., 2014). Cowpea can be grown on a wide variety of soils ranging from predominantly sandy to predominantly clay and $\mathrm{pH}$ range between 4.5 and 8.5.

In Thailand, yardlong bean and cowpea are usually boiled in water resulting in the thick soups and sauces, which are relished. The production sources of Vigna spp. in Thailand are the provinces of Angthong, Nakornpratom, Supanburi, Phichit and Phatthalung (Benchasri et al., 2012). Thailand production areas of yardlong bean and cowpea were estimated at 18,560 - 20,160 ha annually (Sarutayophat et al., 2007). Production output and requirements are currently 
increasing. Production has been increased in order to meet with the higher demand (Benchasri and Bairaman, 2010). Consequently, pesticide has been used in the production process, causing chemical residues in the product and environment (Petlamul et al., 2009; Adeoye et al., 2011; Farahvash and Mirshekari, 2011). Use of pesticide also hikes up the cost of the production process, making it not worth the investment. Nowadays, consumers are paying much more attention to their health (Benchasri, 2015). As a result, vegetable production processes that are safe for both consumers and the environment are even more necessary (Win et al., 2015).

Organic agriculture was established in many countries such as England, Germany, Japan, Thailand and the America (Benchasri, 2015). Organic agricultural emerged in the 1930's and 40's as an alternative to the increasing intensification of agriculture, particularly the use of synthetic nitrogen $(\mathrm{N})$ fertilizers. Sustained high rates of growth in sales of certified organic products in worldwide, averaging $20-25 \%$ per year since 1980, have spurred concomitant growth and activities in production, processing, research, regulation and trade agreements, and exports. The global organic agricultural market value is near $1 \%$ in Thailand, $2 \%$ in the US and $1-5 \%$ in EU countries. Processed organic agricultural have shown particularly rapid growth, often over $100 \%$. Nowadays, commercial certified organic agriculture has spread to over 130 countries worldwide (Lotter, 2003; Reganold and Wachter, 2016). Therefore, the main objectives of this study were to investigate the effects of inorganic and organic agricultural systems in terms of yield, yield components, antioxidant activity and combined analysis gene of Vigna spp. production. The outcome of this study may be used as guidance for yardlong bean and cowpea production. Moreover, we can recommend novelty of work that right varieties should be used for further breeding, physiological as well as antioxidant studies in many countries.

\section{Results}

\section{Physical properties of soil and meteorological data between planting}

The Physio-chemical properties of the soil are given in Table 1. Total nitrogen value in the soil before planting of two systems was low $(0.12 \%$ and $0.12 \%)$. Similarly, the soil had a medium level of phosphorus (33.34 and $37.33 \mathrm{mg} \cdot \mathrm{kg}^{-1}$ ) with a corresponding level of potassium 41.35 and $42.67 \mathrm{mg} \cdot \mathrm{kg}^{-1}$ for before planting under inorganic and organic agricultural systems, respectively. The $\mathrm{pH}$ after planting in water was near neutral about 5.66 and 5.68 in the inorganic and organic agricultural systems, respectively. The present study quantitatively compares the influence of temperature, the level of relative humidity and rainfall on the development times of fifty Vigna spp. genotypes. The meteorological data were shown in Fig 1. air temperatures between January and June 2015 (from $23.14^{\circ} \mathrm{C}$ to $34.80^{\circ} \mathrm{C}$ ). Average air temperature was $28.53^{\circ} \mathrm{C}$. The relative humidity values were between $60.48 \%$ and $95.17 \%$ and the average relative humidity value was $79.06 \%$. Rain day ranged from 3 to 13 days, while the lowest rainfall was recorded in February $5.70 \mathrm{~mm}$.

\section{Fresh pod yield and yield components of Vigna spp.}

Data presented in Table 2 show that the effect of different production was significant of pod length, fresh pod weight, number of seeds.pod ${ }^{-1}$, number of pods.plant ${ }^{-1}$ and fresh pod yield.plant ${ }^{-1}$. Inorganic agricultural system had higher pod number and fresh pod yield than organic agricultural system for all genotypes. The highest number of pods.plant ${ }^{-1}$ was obtained from Fakdang (71.75 \pm 7.27$)$, while the lowest belonged to Taydang $(20.00 \pm 3.37)$. The highest fresh pod yield was recorded from Kangkragan $(1,385.20 \pm 14.40)$. While, yield components on organic agricultural production systems showed that Fakdang was recorded the highest number of pods.plant ${ }^{-1}(55.25 \pm 5.41)$. Kangkragan was observed the highest fresh pod yield.plant ${ }^{-1}(1,135.30 \pm 17.47)$.

\section{Comparison of pod weight, seed numbers, 100 seed weight and harvest index of Vigna spp.}

Figure 2 showed that inorganic agricultural system had higher fresh pod weight, number of seeds, 100 seed weight than organic agricultural system in many genotypes. The most different between production systems for fresh pod weight was Suarpran (11.96 g) (Fig 2A). The number of seeds.pod ${ }^{-1}$ differed production systems between $0.00 \mathrm{~g}$ in Maping and $3.53 \mathrm{~g}$ in Lumtakong (Fig 2B), whereas the differences between production systems for 100 seed weight were between $0.08 \mathrm{~g}$ in Pechkagi and $11.88 \mathrm{~g}$ in Mung 3A (Fig 2C). The final harvest index time was greatly affected by system production. However, many genotypes of Vigna spp. planted under organic agricultural system had time of harvest index more than inorganic agricultural system. The differences between production systems for harvest index were between $0.00 \mathrm{~g}$ in Sansuwan and $18.75 \mathrm{~g}$ in Dawdang (Fig 2D).

\section{Total anthocyanin content (TAC)}

Yardlong bean and cowpea were harvested at edible stage. Dawdang (organic agricultural system) had the highest TAC $\left(603.83 \pm 12.76 \mathrm{mg}\right.$ CGE. $100 \mathrm{~g}^{-1}$ of $\left.\mathrm{FW}\right)$ followed by Kangkragan (480.59 $\pm 9.71 \mathrm{mg}$ CGE. $100 \mathrm{~g}^{-1}$ of FW) and Tuypum (480.43 $\pm 11.65 \mathrm{mg}$ CGE. $100 \mathrm{~g}^{-1}$ of $\left.\mathrm{FW}\right)$, respectively, whereas, Lumnumpong (inorganic agricultural system) had the lowest TAC $\left(30.80 \pm 6.14 \mathrm{mg} \mathrm{CGE} .100 \mathrm{~g}^{-1}\right.$ of FW) (Table 3). Significant differences $(\mathrm{P} \leq 0.05)$ among pod components were observed for TAC.

\section{Total phenolic compound (TPC)}

Significant differences $(\mathrm{P} \leq 0.05)$ among bean genotypes were founded for TPC under inorganic and organic agricultural systems (Table 3). Tuypum (organic agricultural system) had

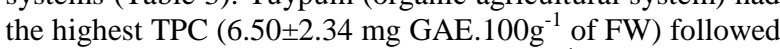
by Fakdang $\left(6.42 \pm 0.24 \mathrm{mg}\right.$ GAE. $100 \mathrm{~g}^{-1}$ of $\left.\mathrm{FW}\right)$ and Dawdang (6.18 $\pm 0.40 \mathrm{mg} \mathrm{GAE} .100 \mathrm{~g}^{-1}$ of FW), respectively, whereas Lumnumpong had the lowest TPC $(2.47 \pm 0.30 \mathrm{mg}$ GAE. $100 \mathrm{~g}^{-1}$ of FW) under organic agricultural system.

\section{Antioxidant capacity}

Differences among yardlong bean and cowpea genotypes planted under inorganic and organic agricultural system were significant $(\mathrm{P} \leq 0.05)$ for parameters related to antioxidant capacity (DPPH, TEAC). The lowest values for TEAC and DPPH were obtained from Taydang and mudkaw 3A (36.16 \pm 1.51 and $2.98 \pm 0.10 \%$ of inhibition, respectively), whereas the highest values were obtained from Panomsarakram and Fakdang $(95.36 \pm 0.29$ and $35.61 \pm 1.46 \%$ of inhibition, respectively) (Table 3). 
Table 1. Physio-chemical properties of the soil $(0-30 \mathrm{~cm})$ of the experimental site.

\begin{tabular}{llllll}
\hline \multirow{2}{*}{ Parameters } & \multicolumn{2}{l}{ Inorganic system } & Organic system & Method of analysis \\
\cline { 2 - 5 } & $\begin{array}{l}\text { Before } \\
\text { planting }\end{array}$ & $\begin{array}{l}\text { After } \\
\text { planting }\end{array}$ & $\begin{array}{l}\text { Before } \\
\text { planting }\end{array}$ & After planting & Walkley-Black method \\
\hline Organic matter $(\%)$ & 1.13 & 1.08 & 1.12 & 1.28 & McKenzie method \\
Nitrogen $(\%)$ & 0.12 & 0.11 & 0.12 & 0.14 & Flame photometric \\
$\mathrm{P}_{2} \mathrm{O}_{5}\left(\mathrm{mg}^{-1} \mathrm{~kg}^{-1}\right)$ & 33.34 & 37.21 & 37.33 & 39.33 & Oxidation \\
$\mathrm{K}\left(\mathrm{mg}_{\mathrm{kg}} \mathrm{kg}^{-1}\right)$ & 41.35 & 41.01 & 42.67 & 44.68 & pH meter method \\
pH $\left(\mathrm{H}_{2} \mathrm{O}\right)$ & 5.67 & 5.66 & 5.67 & 5.68 & \\
EC $\left(\mathrm{dS} \cdot \mathrm{m}^{-1}\right)$ & 0.06 & 0.07 & 0.08 & 0.07 & \\
\hline
\end{tabular}

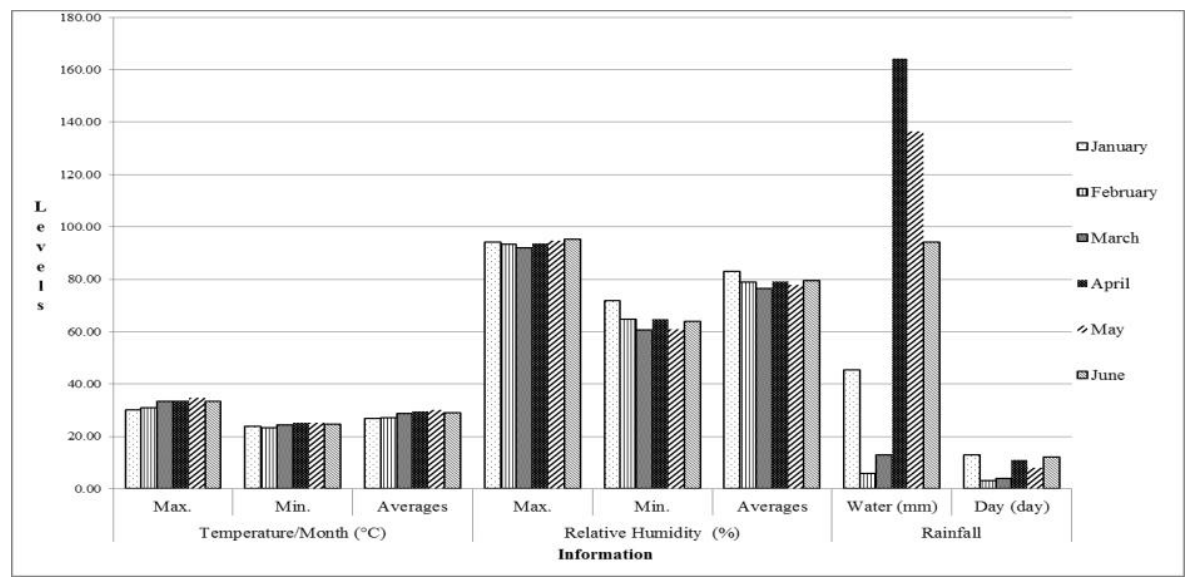

Fig 1. Meteorological data in Phatthalung province, Thailand.

\section{Interaction effect of production systems}

Combined analysis of variance indicated that differences systems (S) and among genotypes (G) were significant of fresh pod length, fresh pod weight, number of pods, fresh pod yield, 100 seed weight and harvest index. There were also significant interactions between system and genotypes ( $\mathrm{S} x$ G) for fresh pod length, fresh pod weight, number of pods, fresh pod yield, 100 seed weight and harvest index for two system plantations. While, the variation of blocks within system for all traits were not significant (Table 4).

\section{Mean comparison}

The mean analysis of fresh pod weights, number of pods, fresh pod yield, 100 seed weight and harvest index are shown that significant differences between system productions in 5 probability level, while pod length and number of seeds were not significant difference (Table 5).

\section{Discussions}

The average monthly temperature ranged from $23.14^{\circ} \mathrm{C}$ to $34.80^{\circ} \mathrm{C}$, while the average relative humidity ranged between $60.48 \%$ and $95.17 \%$. The average monthly temperature, relative humidity and rainfall ranges were considered optimal for the growth and development of Vigna spp. According to Bastos et al. (2002) reported optimum growth and development for Vigna spp. at the temperature of $25-30^{\circ} \mathrm{C}$, while Benchasri and Bairaman (2010) observed an improvement in the performance of yardlong bean with the relative humidity range from $70 \%$ to $85 \%$. Similar results were reported by Ntare (1991). Moreover, soil treatments affect the release of elements (Farahvash and Mirshekari, 2011; Nkaa et al., 2014), day photoperiod, hormone growth and rainy season were also impact on yield and yield components of Vigna spp. (Mukhtar and Singh, 2006).
Yield and yield components of fifty genotypes of Vigna spp. were carried out under agro-chemicals at Thaksin University between January and June 2015, showed differences statistically significant in all genotypes. This is consistent with the reported of Adeoye et al.. (2011). In addition, Nirmal et al. (2001), Magani and Kuchinda (2009), Adeyanju et al. (2012), and Agyeman (2014) reported similar experimental results. However, yield and yield components tested are different in term of terrain and climate (Connell et al., 2015; Karikari et al., 2015). Comparison of fifty Vigna spp. under inorganic and organic farming showed that pod length, number of pods.plant ${ }^{-1}$, number of seeds.pod ${ }^{-1}$, fresh pod yield, 100 seed weight and harvest index showed different statistical significance when tested under the same cultivation agriculture system. Moreover, Fresh pod weight, number of pods.plant ${ }^{-1}, 100$ seed weight, harvest index and fresh pod yield.plant ${ }^{-1}$ were higher under inorganic agricultural system than under organic agricultural system. Similar results were reported by Benchasri (2015). This results, corresponds to the test crops planted under regular (chemical) and under organic fertilizer. It was found that the average yield and yield components test cultivated under inorganic (chemical) rather than organic farming (Attigah et al., 2013) or comparing the yield of green beans is the same effect (Naeem et al., 2006). Moreover, Abbas et al. (2011) reported that some of plant nutrients, when added to the soil in the organic form have low efficiency when comparing to the effect of inorganic fertilizers (Farahvash and Mirshekari, 2011; Olusegun, 2014). In contrast, pod length and number of seeds.pod ${ }^{-1}$ were not significant between systems. Although, yield and yield components of Vigna spp. managed under organic agricultural system were less than inorganic agricultural system, but planting under organic agricultural system was useful for soil improvement and environment conservation (Drinkwater, 2002; Mahoney et al., 2004; 
Table 2. Yield and yield components of Vigna spp. (Mean \pm SE)

\begin{tabular}{|c|c|c|c|c|c|c|c|c|c|c|}
\hline \multirow[b]{2}{*}{ Genotypes } & \multicolumn{2}{|c|}{ Pod length $(\mathrm{cm})$} & \multicolumn{2}{|c|}{ Fresh pod weight.pod-1(g) } & \multicolumn{2}{|c|}{ Number of seeds.pod-1(seed) } & \multicolumn{2}{|c|}{ Number of pods.plant-1 (pod) } & \multicolumn{2}{|c|}{ Fresh pod yield.plant-1(g) } \\
\hline & Inorganic & Organic & Inorganic & Organic & Inorganic & organic & Inorganic & Organic & Inorganic & Organic \\
\hline $28-2$ & $53.06 \pm 4.96 \mathrm{c}-\mathrm{f}$ & $48.19 \pm 10.92 \mathrm{f}-\mathrm{m}$ & $21.37 \pm 0.41 \mathrm{fg}$ & $21.37 \pm 1.12 \mathrm{~cd}$ & $14.60 \pm 1.30 \mathrm{~d}-\mathrm{i}$ & $12.23 \pm 1.12 \mathrm{k}-\mathrm{o}$ & $41.00 \pm 3.74 \mathrm{~b}-\mathrm{i}$ & $33.75 \pm 7.50 \mathrm{~b}-\mathrm{e}$ & $876.20 \pm 29.96 \mathrm{e}-\mathrm{q}$ & $721.20 \pm 10.28 \mathrm{~d}-\mathrm{k}$ \\
\hline 901 & $64.14 \pm 14.08 \mathrm{a}$ & $66.63 \pm 10.10 \mathrm{a}$ & $24.25 \pm 0.16 \mathrm{~d}-\mathrm{e}$ & $24.25 \pm 1.67 \mathrm{bc}$ & $15.78 \pm 4.77 \mathrm{a}-\mathrm{c}$ & $13.18 \pm 1.67 \mathrm{f}-\mathrm{m}$ & $41.00 \pm 6.98 \mathrm{~b}-\mathrm{i}$ & $35.75 \pm 1.56 \mathrm{~b}-\mathrm{d}$ & $994.30 \pm 19.17 \mathrm{c}-\mathrm{m}$ & $866.90 \pm 20.28 \mathrm{a}-\mathrm{h}$ \\
\hline Big 1 & $48.39 \pm 9.91 \mathrm{~d}-\mathrm{k}$ & $47.94 \pm 4.91 \mathrm{~g}-\mathrm{m}$ & $19.93 \pm 0.35 \mathrm{f}-\mathrm{h}$ & $19.93 \pm 0.65 \mathrm{de}$ & $13.58 \pm 5.80 \mathrm{~h}-\mathrm{m}$ & $14.85 \pm 2.65 \mathrm{~b}-\mathrm{f}$ & $38.25 \pm 7.27 \mathrm{~b}-\mathrm{i}$ & $39.00 \pm 6.48 \mathrm{a}-\mathrm{d}$ & $762.30 \pm 14.98 \mathrm{i}-\mathrm{r}$ & $777.30 \pm 19.16 \mathrm{~b}-\mathrm{j}$ \\
\hline Dawdang & $52.84 \pm 5.52 \mathrm{c}-\mathrm{f}$ & $45.52 \pm 6.71 \mathrm{~h}-\mathrm{o}$ & $16.33 \pm 0.05 \mathrm{~h}$ & $14.72 \pm 0.20 \mathrm{~h}$ & $13.95 \pm 4.04 \mathrm{~d}-1$ & $14.45 \pm 4.20 \mathrm{~d}-\mathrm{h}$ & $36.75 \pm 6.02 \mathrm{~d}-\mathrm{j}$ & $31.25 \pm 7.80 \mathrm{~b}-\mathrm{e}$ & $600.10 \pm 98.32 \mathrm{q}-\mathrm{u}$ & $460.00 \pm 19.82 \mathrm{j}-\mathrm{m}$ \\
\hline Dokkrong 10 & $41.80 \pm 10.01 \mathrm{i}-\mathrm{n}$ & $45.79 \pm 7.61 \mathrm{~h}-\mathrm{n}$ & $10.72 \pm 0.211$ & $10.6 \pm 0.85 \mathrm{i}$ & $14.33 \pm 5.90 \mathrm{~d}-\mathrm{j}$ & $13.65 \pm 2.85 \mathrm{e}-1$ & $30.75 \pm 1.12 \mathrm{~g}-\mathrm{k}$ & $24.00 \pm 5.48 \mathrm{~d}-\mathrm{g}$ & $329.60 \pm 55.48 \mathrm{v}$ & $254.40 \pm 14.41 \mathrm{n}$ \\
\hline Ero 6 & $46.30 \pm 5.59 \mathrm{e}-\mathrm{m}$ & $60.33 \pm 10.80 \mathrm{a}-\mathrm{d}$ & $22.71 \pm 0.52 \mathrm{e}-\mathrm{g}$ & $22.71 \pm 1.11 \mathrm{c}-\mathrm{d}$ & $13.38 \pm 3.34 \mathrm{i}-\mathrm{m}$ & $12.58 \pm 4.11 \mathrm{k}-\mathrm{n}$ & $51.50 \pm 2.07 \mathrm{~b}-\mathrm{d}$ & $42.50 \pm 3.70 \mathrm{a}-\mathrm{d}$ & $1169.60 \pm 24.09 \mathrm{a}-\mathrm{f}$ & $965.20 \pm 83.96 \mathrm{a}-\mathrm{c}$ \\
\hline Fakdang* & $26.91 \pm 1.73 \mathrm{pq}$ & $24.80 \pm 2.71 \mathrm{~s}$ & $12.38 \pm 0.42 \mathrm{k}$ & $12.38 \pm 0.84 \mathrm{hi}$ & $14.25 \pm 6.45 \mathrm{~d}-\mathrm{j}$ & $15.23 \pm 4.84 \mathrm{~b}-\mathrm{e}$ & $71.75 \pm 7.27 \mathrm{a}$ & $55.25 \pm 5.41 \mathrm{a}$ & $888.30 \pm 36.51 \mathrm{e}-\mathrm{q}$ & $684.00 \pm 90.06 \mathrm{e}-\mathrm{k}$ \\
\hline Fakkeal* & $40.11 \pm 6.251-n$ & $37.20 \pm 5.47 \mathrm{n}-\mathrm{q}$ & $19.06 \pm 0.63 \mathrm{f}-\mathrm{h}$ & $19.06 \pm 0.74 \mathrm{de}$ & $16.25 \pm 4.05 \mathrm{ab}$ & $14.88 \pm 5.74 \mathrm{~b}-\mathrm{f}$ & $35.00 \pm 4.24 \mathrm{e}-\mathrm{k}$ & $30.00 \pm 5.94 \mathrm{~b}-\mathrm{f}$ & $667.10 \pm 80.86 \mathrm{~m}-\mathrm{s}$ & $571.80 \pm 13.3 \mathrm{~g}-\mathrm{m}$ \\
\hline Green aro & $47.29 \pm 9.33 \mathrm{e}-1$ & $47.25 \pm 5.36 \mathrm{~h}-\mathrm{m}$ & $23.63 \pm 0.82 \mathrm{ef}$ & $23.52 \pm 0.31 \mathrm{~b}-\mathrm{d}$ & $13.68 \pm 2.69 \mathrm{~g}-\mathrm{m}$ & $13.83 \pm 3.30 \mathrm{e}-\mathrm{k}$ & $43.50 \pm 8.77 \mathrm{~b}-\mathrm{h}$ & $40.00 \pm 7.02 \mathrm{a}-\mathrm{d}$ & $1027.70 \pm 26.01 \mathrm{~b}-\mathrm{k}$ & $940.60 \pm 16.32 \mathrm{a}-\mathrm{f}$ \\
\hline Green aro $692^{*}$ & $50.56 \pm 8.48 \mathrm{~d}-\mathrm{h}$ & $42.95 \pm 11.16 \mathrm{k}-\mathrm{p}$ & $14.34 \pm 0.18 \mathrm{j}$ & $12.9 \pm 0.57 \mathrm{hi}$ & $15.38 \pm 3.59 \mathrm{a}-\mathrm{f}$ & $13.45 \pm 5.57 \mathrm{f}-1$ & $49.25 \pm 3.86 \mathrm{~b}-\mathrm{e}$ & $46.50 \pm 3.11 \mathrm{ab}$ & $706.40 \pm 78.54 \mathrm{k}-\mathrm{s}$ & $799.80 \pm 53.48 \mathrm{~b}-\mathrm{i}$ \\
\hline Hero* & $44.94 \pm 7.78 \mathrm{~g}-\mathrm{n}$ & $41.40 \pm 9.001-\mathrm{p}$ & $16.08 \pm 0.33 \mathrm{hi}$ & $13.7 \pm 0.07 \mathrm{hi}$ & $13.48 \pm 5.91 \mathrm{~h}-\mathrm{m}$ & $11.45 \pm 1.07 n-p$ & $41.00 \pm 5.48 \mathrm{~b}-\mathrm{i}$ & $34.00 \pm 6.27 \mathrm{~b}-\mathrm{e}$ & $659.10 \pm 14.73 \mathrm{n}-\mathrm{s}$ & $465.80 \pm 18.06 \mathrm{i}-\mathrm{n}$ \\
\hline Jawsamran & $47.96 \pm 11.54 \mathrm{~d}-\mathrm{k}$ & $47.09 \pm 10.93 \mathrm{~h}-\mathrm{m}$ & $25.98 \pm 0.64 \mathrm{c}-\mathrm{e}$ & $25.98 \pm 0.13 b$ & $12.68 \pm 1.84 \mathrm{k}-\mathrm{m}$ & $13.88 \pm 6.13 \mathrm{~d}-\mathrm{k}$ & $44.25 \pm 6.50 \mathrm{~b}-\mathrm{h}$ & $31.25 \pm 2.25 \mathrm{~b}-\mathrm{e}$ & $1149.60 \pm 18.87 \mathrm{a}-\mathrm{g}$ & $811.90 \pm 20.34 \mathrm{a}-\mathrm{i}$ \\
\hline Jiandou & $58.97 \pm 5.23 \mathrm{a}-\mathrm{c}$ & $49.70 \pm 9.57 \mathrm{e}-1$ & $24.42 \pm 0.87 \mathrm{de}$ & $24.42 \pm 0.05 \mathrm{bc}$ & $14.70 \pm 4.29 \mathrm{~d}-\mathrm{i}$ & $13.88 \pm 1.44 \mathrm{~d}-\mathrm{k}$ & $51.50 \pm 6.24 \mathrm{~b}-\mathrm{d}$ & $42.50 \pm 5.69 \mathrm{a}-\mathrm{d}$ & $1257.60 \pm 12.50 \mathrm{a}-\mathrm{d}$ & $1037.90 \pm 18.86 \mathrm{a}-\mathrm{d}$ \\
\hline $\operatorname{Jin} 6$ & $59.02 \pm 10.61 \mathrm{a}-\mathrm{c}$ & $63.18 \pm 11.95 \mathrm{a}-\mathrm{c}$ & $28.03 \pm 0.10 \mathrm{bc}$ & $28.03 \pm 0.50 \mathrm{a}$ & $15.68 \pm 3.68 \mathrm{a}-\mathrm{c}$ & $15.73 \pm 3.50 \mathrm{bc}$ & $44.75 \pm 8.77 \mathrm{~b}-\mathrm{h}$ & $39.00 \pm 4.83 \mathrm{a}-\mathrm{d}$ & $1254.30 \pm 25.83 \mathrm{a}-\mathrm{d}$ & $1093.20 \pm 15.40 \mathrm{a}-\mathrm{b}$ \\
\hline Jin 8 & $64.81 \pm 10.02 \mathrm{a}$ & $63.64 \pm 7.81 \mathrm{ab}$ & $21.04 \pm 0.25 \mathrm{fg}$ & $18.2 \pm 0.79 \mathrm{eg}$ & $15.28 \pm 3.13 \mathrm{a}-\mathrm{f}$ & $16.30 \pm 2.79 \mathrm{ab}$ & $48.50 \pm 8.06 \mathrm{~b}-\mathrm{f}$ & $30.50 \pm 8.58 \mathrm{~b}-\mathrm{e}$ & $1020.50 \pm 10.41 \mathrm{~b}-1$ & $555.10 \pm 13.93 \mathrm{~g}-\mathrm{n}$ \\
\hline Jong ang & $46.18 \pm 8.65 \mathrm{f}-\mathrm{m}$ & $52.46 \pm 9.28 \mathrm{~d}-\mathrm{j}$ & $12.16 \pm 0.09 \mathrm{k}$ & $12.68 \pm 0.38 \mathrm{hi}$ & $14.35 \pm 2.59 \mathrm{~d}-\mathrm{j}$ & $12.38 \pm 5.38 \mathrm{ko}$ & $39.50 \pm 9.88 \mathrm{~b}-\mathrm{i}$ & $36.75 \pm 3.77 \mathrm{~b}-\mathrm{d}$ & $559.30 \pm 89.04 \mathrm{q}-\mathrm{u}$ & $466.00 \pm 58.13 \mathrm{i}-\mathrm{n}$ \\
\hline Kangkragan & $48.39 \pm 6.56 \mathrm{~d}-\mathrm{k}$ & $36.74 \pm 6.230-q$ & $28.56 \pm 0.08 \mathrm{bc}$ & $28.56 \pm 0.24 \mathrm{a}$ & $14.73 \pm 2.66 \mathrm{~b}-\mathrm{i}$ & $14.35 \pm 2.24 \mathrm{~d}-\mathrm{h}$ & $48.50 \pm 6.81 \mathrm{~b}-\mathrm{f}$ & $39.75 \pm 4.11 \mathrm{a}-\mathrm{d}$ & $1385.20 \pm 14.40 \mathrm{a}$ & $1135.30 \pm 17.47 \mathrm{a}$ \\
\hline Kaset $135^{*}$ & $41.31 \pm 6.85 \mathrm{j}-\mathrm{n}$ & $54.38 \pm 10.17 \mathrm{~d}-\mathrm{i}$ & $18.15 \pm 0.07 \mathrm{f}-\mathrm{h}$ & $16.2 \pm 0.14 \mathrm{fg}$ & $13.68 \pm 4.19 \mathrm{~g}-\mathrm{m}$ & $13.18 \pm 3.14 \mathrm{f}-\mathrm{m}$ & $40.75 \pm 3.82 \mathrm{~b}-\mathrm{i}$ & $32.50 \pm 1.66 \mathrm{~b}-\mathrm{e}$ & $739.60 \pm 26.09 \mathrm{j}-\mathrm{s}$ & $526.60 \pm 25.55 \mathrm{i}-\mathrm{n}$ \\
\hline keawdok 4 & $55.34 \pm 10.25 \mathrm{~b}-\mathrm{d}$ & $57.58 \pm 12.75 \mathrm{~b}-\mathrm{e}$ & $21.25 \pm 0.40 \mathrm{fg}$ & $21.25 \pm 0.65 \mathrm{c}-\mathrm{e}$ & $14.98 \pm 2.56 \mathrm{~b}-\mathrm{h}$ & $12.70 \pm 3.65 \mathrm{i}-\mathrm{n}$ & $40.50 \pm 2.01 \mathrm{~b}-\mathrm{i}$ & $38.75 \pm 7.80 \mathrm{a}-\mathrm{d}$ & $860.60 \pm 25.29 \mathrm{f}-\mathrm{q}$ & $823.40 \pm 65.85 \mathrm{a}-\mathrm{i}$ \\
\hline Longgo 5 & $63.75 \pm 10.29 \mathrm{a}$ & $60.85 \pm 7.86 \mathrm{a}-\mathrm{d}$ & $24.59 \pm 0.05 \mathrm{de}$ & $24.28 \pm 0.40 \mathrm{bc}$ & $14.08 \pm 4.86 \mathrm{~d}-\mathrm{k}$ & $14.35 \pm 1.47 \mathrm{~d}-\mathrm{i}$ & $36.50 \pm 3.32 \mathrm{~d}-\mathrm{j}$ & $29.50 \pm 3.70 \mathrm{~b}-\mathrm{f}$ & $897.50 \pm 81.56 \mathrm{e}-\mathrm{p}$ & $716.40 \pm 87.76 \mathrm{~d}-\mathrm{k}$ \\
\hline Lumnumchee & $41.47 \pm 5.60 \mathrm{i}-\mathrm{n}$ & $50.54 \pm 10.86 \mathrm{e}-1$ & $22.27 \pm 0.32 \mathrm{eg}$ & $22.13 \pm 0.51 \mathrm{c}-\mathrm{e}$ & $14.28 \pm 4.36 \mathrm{~d}-\mathrm{j}$ & $14.55 \pm 4.51 \mathrm{~d}-\mathrm{g}$ & $36.01 \pm 8.40 \mathrm{~d}-\mathrm{j}$ & $20.50 \pm 2.08 \mathrm{~d}-\mathrm{g}$ & $801.90 \pm 19.84 \mathrm{~h}-\mathrm{r}$ & $453.60 \pm 6.44 \mathrm{j}-\mathrm{m}$ \\
\hline Lumnumpong & $48.97 \pm 4.20 \mathrm{~d}-\mathrm{i}$ & $51.14 \pm 7.48 \mathrm{e}-\mathrm{k}$ & $27.93 \pm 0.29 \mathrm{bd}$ & $24.86 \pm 0.24 \mathrm{bc}$ & $12.93 \pm 5.12 \mathrm{j}-\mathrm{m}$ & $13.83 \pm 3.50 \mathrm{~d}-\mathrm{k}$ & $40.50 \pm 1.90 \mathrm{~b}-\mathrm{i}$ & $34.00 \pm 5.60 \mathrm{~b}-\mathrm{e}$ & $1131.10 \pm 20.66 \mathrm{a}-\mathrm{h}$ & $845.20 \pm 39.16 \mathrm{a}-\mathrm{h}$ \\
\hline Lumtakong & $25.44 \pm 6.40 \mathrm{pq}$ & $26.24 \pm 5.49 \mathrm{~s}$ & $15.5 \pm 0.28 \mathrm{i}$ & $10.83 \pm 0.21 \mathrm{i}$ & $14.08 \pm 3.59 \mathrm{~d}-\mathrm{k}$ & $10.55 \pm 1.20 \mathrm{q}$ & $39.25 \pm 2.09 \mathrm{~b}-\mathrm{i}$ & $36.25 \pm 7.72 \mathrm{~b}-\mathrm{d}$ & $608.30 \pm 12.19 p-u$ & $392.60 \pm 83.6 \mathrm{k}-\mathrm{n}$ \\
\hline Maping & $37.54 \pm 14.93 \mathrm{no}$ & $49.64 \pm 9.87 \mathrm{e}-1$ & $25.48 \pm 0.64 \mathrm{c}-\mathrm{e}$ & $19.70 \pm 0.65 \mathrm{de}$ & $14.30 \pm 3.65 \mathrm{~d}-\mathrm{j}$ & $14.30 \pm 3.65 \mathrm{~d}-\mathrm{j}$ & $41.50 \pm 1.33 \mathrm{~b}-\mathrm{i}$ & $37.35 \pm 7.09 \mathrm{~b}-\mathrm{d}$ & $1057.40 \pm 18.07 a-j$ & $735.70 \pm 40 \mathrm{~d}-\mathrm{k}$ \\
\hline MMS1* & $53.84 \pm 10.52 \mathrm{~b}-\mathrm{e}$ & $42.96 \pm 7.76 \mathrm{k}-\mathrm{p}$ & $16.71 \pm 0.40 \mathrm{~h}$ & $14.01 \pm 0.92 \mathrm{hi}$ & $12.53 \pm 1.51-\mathrm{n}$ & $14.75 \pm 1.92 \mathrm{~b}-\mathrm{f}$ & $43.50 \pm 4.42 \mathrm{~b}-\mathrm{h}$ & $36.01 \pm 9.49 \mathrm{~b}-\mathrm{d}$ & $727.00 \pm 15.49 \mathrm{j}-\mathrm{s}$ & $504.40 \pm 59.28 \mathrm{i}-\mathrm{n}$ \\
\hline mudkaw $3 \mathrm{~A}^{*}$ & $46.69 \pm 7.60 \mathrm{e}-\mathrm{m}$ & $50.40 \pm 7.85 \mathrm{e}-1$ & $21.45 \pm 0.37 \mathrm{fg}$ & $16.78 \pm 0.28 \mathrm{fg}$ & $11.05 \pm 6.55 \mathrm{no}$ & $12.50 \pm 2.28 \mathrm{k}-\mathrm{n}$ & $36.50 \pm 8.27 \mathrm{~d}-\mathrm{j}$ & $40.75 \pm 7.80 \mathrm{a}-\mathrm{d}$ & $782.90 \pm 17.31 \mathrm{i}-\mathrm{r}$ & $683.60 \pm 67.42 \mathrm{e}-\mathrm{k}$ \\
\hline Mung 3A & $43.38 \pm 8.16 \mathrm{~h}-\mathrm{n}$ & $41.72 \pm 7.021-\mathrm{p}$ & $22.03 \pm 0.21 \mathrm{e}-\mathrm{g}$ & $21.99 \pm 0.27 \mathrm{c}-\mathrm{e}$ & $15.13 \pm 5.74 \mathrm{~b}-\mathrm{h}$ & $14.33 \pm 4.27 \mathrm{~d}-\mathrm{j}$ & $37.25 \pm 7.63 \mathrm{~d}-\mathrm{i}$ & $32.05 \pm 9.06 \mathrm{~b}-\mathrm{e}$ & $820.60 \pm 18.14 \mathrm{~g}-\mathrm{r}$ & $704.90 \pm 19.51 \mathrm{~d}-\mathrm{k}$ \\
\hline $\begin{array}{l}\text { mungsitid } \\
\text { mus }\end{array}$ & $37.94 \pm 5.44 \mathrm{no}$ & $48.52 \pm 5.04 \mathrm{f}-\mathrm{m}$ & $17.48 \pm 0.58 \mathrm{gh}$ & $16.25 \pm 0.34 \mathrm{fg}$ & $13.35 \pm 4.58 \mathrm{i}-\mathrm{m}$ & $12.48 \pm 2.34 \mathrm{k}-\mathrm{n}$ & $39.50 \pm 7.51 \mathrm{~b}-\mathrm{i}$ & $20.00 \pm 3.48 \mathrm{~d}-\mathrm{g}$ & $69.40 \pm 11.571-\mathrm{s}$ & $325.02 \pm 36.281-\mathrm{n}$ \\
\hline $\mathrm{Neu}$ & $28.95 \pm 3.24 \mathrm{p}$ & $45.74 \pm 11.09 \mathrm{~h}-\mathrm{n}$ & $26.03 \pm 0.28 \mathrm{~cd}$ & $25.93 \pm 0.26 \mathrm{~b}$ & $13.93 \pm 5.91 \mathrm{e}-1$ & $13.00 \pm 1.26 \mathrm{~g}-\mathrm{n}$ & $29.25 \pm 0.21 \mathrm{~h}-\mathrm{k}$ & $12.05 \pm 3.16 \mathrm{~g}$ & $761.40 \pm 25.77 \mathrm{i}-\mathrm{r}$ & $312.40 \pm 82.311-\mathrm{n}$ \\
\hline Nhongsau & $48.56 \pm 11.11 \mathrm{~d}-\mathrm{j}$ & $42.97 \pm 8.76 \mathrm{k}-\mathrm{p}$ & $25.19 \pm 0.18 \mathrm{c}-\mathrm{e}$ & $25.26 \pm 0.04 \mathrm{~b}$ & $16.55 \pm 6.35 \mathrm{a}$ & $15.58 \pm 2.04 \mathrm{~b}-\mathrm{d}$ & $53.50 \pm 5.45 \mathrm{bc}$ & $37.25 \pm 9.57 \mathrm{~b}-\mathrm{d}$ & $1347.70 \pm 34.58 \mathrm{ab}$ & $940.90 \pm 41.74 \mathrm{a}-\mathrm{f}$ \\
\hline Nigro & $55.18 \pm 5.29 \mathrm{~b}-\mathrm{d}$ & $54.64 \pm 10.60 \mathrm{c}-\mathrm{h}$ & $18.73 \pm 0.87 \mathrm{fh}$ & $18.73 \pm 0.68 \mathrm{e}-\mathrm{g}$ & $13.70 \pm 6.06 \mathrm{~g}-\mathrm{m}$ & $11.48 \pm 5.68 \mathrm{~m}-\mathrm{p}$ & $38.75 \pm 3.82 \mathrm{~b}-\mathrm{i}$ & $36.50 \pm 7.10 \mathrm{~b}-\mathrm{d}$ & $725.80 \pm 28.80 \mathrm{j}-\mathrm{s}$ & $683.60 \pm 30.24 \mathrm{e}-\mathrm{k}$ \\
\hline Nuangtong9 & $48.39 \pm 4.57 \mathrm{~d}-\mathrm{k}$ & $45.56 \pm 10.64 \mathrm{~h}-\mathrm{o}$ & $22.63 \pm 0.60 \mathrm{eg}$ & $22.60 \pm 0.80 \mathrm{c}-\mathrm{e}$ & $14.40 \pm 4.90 \mathrm{~d}-\mathrm{j}$ & $14.53 \pm 6.80 \mathrm{~d}-\mathrm{g}$ & $37.75 \pm 1.05 \mathrm{c}-\mathrm{i}$ & $32.05 \pm 1.36 \mathrm{~b}-\mathrm{e}$ & $854.30 \pm 27.33 \mathrm{f}-\mathrm{q}$ & $724.20 \pm 34.45 \mathrm{~d}-\mathrm{k}$ \\
\hline Panomsarakram & $42.27 \pm 12.42 \mathrm{i}-\mathrm{n}$ & $49.81 \pm 7.61 \mathrm{e}-1$ & $21.17 \pm 0.30 \mathrm{fg}$ & $21.17 \pm 1.75 \mathrm{c}-\mathrm{e}$ & $12.63 \pm 8.50 \mathrm{k}-\mathrm{m}$ & $13.65 \pm 1.75 \mathrm{e}-1$ & $32.50 \pm 7.42 \mathrm{f}-\mathrm{k}$ & $29.25 \pm 8.18 \mathrm{c}-\mathrm{f}$ & $688.00 \pm 57.101-\mathrm{s}$ & $619.20 \pm 98.35 \mathrm{f}-\mathrm{m}$ \\
\hline Pechkagi & $44.46 \pm 6.91 \mathrm{~g}-\mathrm{n}$ & $43.23 \pm 7.93 \mathrm{j}-\mathrm{p}$ & $18.00 \pm 0.53 \mathrm{fh}$ & $16.48 \pm 0.46 \mathrm{fg}$ & $13.53 \pm 4.19 \mathrm{~h}-\mathrm{m}$ & $13.58 \pm 3.46 \mathrm{e}-1$ & $38.25 \pm 7.10 \mathrm{~b}-\mathrm{i}$ & $32.00 \pm 8.41 \mathrm{~b}-\mathrm{e}$ & $688.60 \pm 13.331-\mathrm{s}$ & $527.40 \pm 38.54 \mathrm{~h}-\mathrm{n}$ \\
\hline Pechpimai* & $28.19 \pm 3.08 \mathrm{p}$ & $23.53 \pm 3.41 \mathrm{~s}$ & $17.78 \pm 0.38 \mathrm{gh}$ & $17.49 \pm 0.74 \mathrm{e}-\mathrm{g}$ & $14.98 \pm 3.50 \mathrm{~b}-\mathrm{h}$ & $17.48 \pm 5.74 \mathrm{a}$ & $42.25 \pm 3.77 \mathrm{~b}-\mathrm{h}$ & $35.00 \pm 7.44 \mathrm{~b}-\mathrm{d}$ & $751.20 \pm 67.12 \mathrm{j}-\mathrm{s}$ & $612.30 \pm 32.26 \mathrm{f}-\mathrm{m}$ \\
\hline Raya & $39.39 \pm 16.59 \mathrm{mn}$ & $56.57 \pm 7.94 \mathrm{~b}-\mathrm{g}$ & $23.38 \pm 0.62 \mathrm{ef}$ & $23.38 \pm 1.04 \mathrm{~b}-\mathrm{d}$ & $12.30 \pm 4.97 \mathrm{mn}$ & $13.40 \pm 4.04 \mathrm{f}-1$ & $46.00 \pm 1.61 \mathrm{~b}-\mathrm{g}$ & $40.25 \pm 5.85 \mathrm{a}-\mathrm{d}$ & $1075.50 \pm 48.17 \mathrm{a}-\mathrm{i}$ & $941.00 \pm 36.83 \mathrm{a}-\mathrm{f}$ \\
\hline Samchuk & $60.77 \pm 7.46 \mathrm{a}$ & $57.23 \pm 11.19 \mathrm{~b}-\mathrm{f}$ & $21.64 \pm 0.16 \mathrm{fg}$ & $21.62 \pm 0.67 \mathrm{c}-\mathrm{e}$ & $14.05 \pm 4.06 \mathrm{~d}-1$ & $12.63 \pm 1.67 \mathrm{j}-\mathrm{n}$ & $45.50 \pm 1.08 \mathrm{~b}-\mathrm{g}$ & $25.00 \pm 2.96 \mathrm{~d}-\mathrm{g}$ & $984.40 \pm 37.19 \mathrm{~d}-\mathrm{n}$ & $543.80 \pm 31.43 \mathrm{~g}-\mathrm{n}$ \\
\hline Sansuwan & $58.86 \pm 5.75 \mathrm{a}-\mathrm{c}$ & $60.54 \pm 11.65 \mathrm{a}-\mathrm{d}$ & $26.65 \pm 0.41 \mathrm{~cd}$ & $25.18 \pm 0.33 \mathrm{~b}$ & $15.48 \pm 4.89 \mathrm{a}-\mathrm{d}$ & $12.78 \pm 3.30 \mathrm{~h}-\mathrm{n}$ & $49.75 \pm 4.43 \mathrm{~b}-\mathrm{e}$ & $37.75 \pm 1.05 \mathrm{~b}-\mathrm{d}$ & $1325.60 \pm 21.87 \mathrm{a}-\mathrm{c}$ & $950.60 \pm 79.55 \mathrm{a}-\mathrm{c}$ \\
\hline Sayrung & $39.77 \pm 7.041-\mathrm{n}$ & $47.33 \pm 8.40 \mathrm{~h}-\mathrm{m}$ & $9.66 \pm 0.58 \mathrm{~m}$ & $9.05 \pm 0.38 \mathrm{j}$ & $13.78 \pm 4.03 \mathrm{f}-\mathrm{m}$ & $11.58 \pm 3.38 \mathrm{~m}-\mathrm{p}$ & $44.00 \pm 8.33 \mathrm{~b}-\mathrm{h}$ & $38.75 \pm 4.57 \mathrm{a}-\mathrm{d}$ & $425.10 \pm 30.97 \mathrm{~s}-\mathrm{u}$ & $350.50 \pm 68.511-\mathrm{n}$ \\
\hline Saytan & $44.99 \pm 5.23 \mathrm{~g}-\mathrm{n}$ & $50.59 \pm 7.09 \mathrm{e}-1$ & $22.18 \pm 0.28 \mathrm{eg}$ & $18.96 \pm 0.31 \mathrm{e}-\mathrm{g}$ & $14.43 \pm 3.74 \mathrm{~d}-\mathrm{j}$ & $12.55 \pm 2.26 \mathrm{k}-\mathrm{n}$ & $45.75 \pm 4.99 \mathrm{~b}-\mathrm{g}$ & $41.00 \pm 1.39 \mathrm{a}-\mathrm{d}$ & $1014.80 \pm 10.32 \mathrm{~b}-1$ & $777.40 \pm 17.04 \mathrm{~b}-\mathrm{j}$ \\
\hline Saytara & $53.56 \pm 8.27 \mathrm{~b}-\mathrm{e}$ & $49.80 \pm 7.11 \mathrm{e}-1$ & $24.32 \pm 0.59 \mathrm{de}$ & $24.32 \pm 0.65 \mathrm{bc}$ & $10.75 \pm 2.71 \mathrm{o}$ & $13.00 \pm 3.65 \mathrm{f}-\mathrm{m}$ & $26.25 \pm 7.27 \mathrm{i}-\mathrm{k}$ & $26.75 \pm 1.34 \mathrm{~d}-\mathrm{g}$ & $638.40 \pm 76.910-\mathrm{u}$ & $650.60 \pm 51.47 \mathrm{e}-1$ \\
\hline Saytip & $43.02 \pm 9.91 \mathrm{~h}-\mathrm{n}$ & $45.88 \pm 10.06 \mathrm{~h}-\mathrm{n}$ & $8.45 \pm 0.89 n$ & $8.28 \pm 0.42 \mathrm{k}$ & $14.70 \pm 5.34 \mathrm{~d}-\mathrm{i}$ & $13.50 \pm 1.42 \mathrm{f}-1$ & $49.25 \pm 5.97 \mathrm{~b}-\mathrm{e}$ & $42.00 \pm 4.39 \mathrm{a}-\mathrm{d}$ & $416.20 \pm 67.40 \mathrm{tu}$ & $347.80 \pm 57.511-\mathrm{n}$ \\
\hline Suarpran* & $19.77 \pm 4.93 q$ & $22.36 \pm 3.57 \mathrm{~s}$ & $31.78 \pm 1.58 \mathrm{bc}$ & $19.82 \pm 0.80 \mathrm{de}$ & $13.38 \pm 3.59 \mathrm{i}-\mathrm{m}$ & $10.75 \pm 2.80 \mathrm{o}-\mathrm{q}$ & $21.00 \pm 8.45 \mathrm{jk}$ & $10.50 \pm 3.11 \mathrm{~g}$ & $667.40 \pm 94.64 \mathrm{~m}-\mathrm{s}$ & $208.10 \pm 61.620$ \\
\hline Taydang* & $28.93 \pm 9.87 \mathrm{p}$ & $34.91 \pm 1.59 \mathrm{p}-\mathrm{r}$ & $33.57 \pm 1.48 \mathrm{a}$ & $28.17 \pm 0.54 a$ & $13.88 \pm 2.96 \mathrm{e}-1$ & $13.58 \pm 2.54 \mathrm{e}-1$ & $20.00 \pm 3.37 \mathrm{k}$ & $17.25 \pm 5.32 \mathrm{e}-\mathrm{g}$ & $671.40 \pm 13.64 \mathrm{~m}-\mathrm{s}$ & $486.00 \pm 88.12 \mathrm{i}-\mathrm{n}$ \\
\hline Tuy ngu & $31.41 \pm 7.96 \mathrm{op}$ & $30.73 \pm 4.87 \mathrm{q}-\mathrm{r}$ & $23.28 \pm 0.18 \mathrm{ef}$ & $23.28 \pm 1.47 \mathrm{~b}-\mathrm{d}$ & $8.77 \pm 4.36 \mathrm{p}$ & $9.65 \pm 1.47 \mathrm{q}$ & $21.25 \pm 2.06 \mathrm{jk}$ & $13.00 \pm 3.92 \mathrm{f}-\mathrm{g}$ & $494.70 \pm 47.99 \mathrm{r}-\mathrm{u}$ & $302.60 \pm 91.61 \mathrm{mn}$ \\
\hline Tuypum* & $30.69 \pm 4.53 \mathrm{op}$ & $27.48 \pm 5.28 \mathrm{rs}$ & $16.75 \pm 0.27 \mathrm{~h}$ & $16.4 \pm 0.80 \mathrm{fg}$ & $13.43 \pm 3.38 \mathrm{i}-\mathrm{m}$ & $13.38 \pm 2.80 \mathrm{f}-1$ & $39.75 \pm 1.69 \mathrm{~b}-\mathrm{i}$ & $38.05 \pm 2.73 \mathrm{~b}-\mathrm{d}$ & $665.80 \pm 79.04 \mathrm{~m}-\mathrm{s}$ & $624.20 \pm 34.21 \mathrm{el}$ \\
\hline Tuysan & $51.79 \pm 7.81 \mathrm{c}-\mathrm{g}$ & $53.77 \pm 8.60 \mathrm{~d}-\mathrm{i}$ & $20.3 \pm 0.13 \mathrm{fh}$ & $17.75 \pm 0.59 \mathrm{e}-\mathrm{g}$ & $14.08 \pm 4.03 \mathrm{~d}-\mathrm{k}$ & $12.88 \pm 3.59 \mathrm{~g}-\mathrm{n}$ & $47.75 \pm 2.54 \mathrm{~b}-\mathrm{f}$ & $37.25 \pm 4.82 \mathrm{~b}-\mathrm{d}$ & $969.30 \pm 93.56 \mathrm{~d}-\mathrm{o}$ & $661.30 \pm 30.87 \mathrm{el}$ \\
\hline Violet 696 & $40.91 \pm 5.991-\mathrm{n}$ & $39.36 \pm 8.58 \mathrm{~m}-\mathrm{p}$ & $17.75 \pm 0.28 \mathrm{gh}$ & $16.00 \pm 0.47 \mathrm{~g}$ & $14.38 \pm 4.03 \mathrm{~d}-\mathrm{j}$ & $11.95 \pm 1.701-p$ & $47.75 \pm 5.68 \mathrm{~b}-\mathrm{f}$ & $44.50 \pm 5.26 \mathrm{a}-\mathrm{c}$ & $847.40 \pm 94.37 \mathrm{f}-\mathrm{q}$ & $712.10 \pm 95.99 \mathrm{~d}-\mathrm{i}$ \\
\hline Winggao OP & $43.48 \pm 10.21 \mathrm{~h}-\mathrm{n}$ & $45.22 \pm 7.41 \mathrm{i}-\mathrm{O}$ & $24.64 \pm 1.13 \mathrm{de}$ & $23.06 \pm 0.35 \mathrm{~b}-\mathrm{d}$ & $13.65 \pm 6.66 \mathrm{~g}-\mathrm{m}$ & $11.40 \pm 5.35 n-p$ & $48.50 \pm 3.70 \mathrm{~b}-\mathrm{f}$ & $44.25 \pm 1.47 \mathrm{a}-\mathrm{c}$ & $1195.00 \pm 91.09 \mathrm{a}-\mathrm{d}$ & $1020.30 \pm 21.36 \mathrm{ab}$ \\
\hline Yokkhaw & $42.02 \pm 10.13 \mathrm{i}-\mathrm{n}$ & $46.28 \pm 14.39 \mathrm{~h}-\mathrm{m}$ & $19.5 \pm 0.26 \mathrm{fh}$ & $19.63 \pm 0.93 \mathrm{e}$ & $14.60 \pm 4.16 \mathrm{~d}-\mathrm{i}$ & $12.93 \pm 1.93 \mathrm{~g}-\mathrm{n}$ & $53.75 \pm 3.90 \mathrm{~b}-\mathrm{f}$ & $45.02 \pm 2.94 \mathrm{a}-\mathrm{c}$ & $1048.10 \pm 75.41 \mathrm{~b}-\mathrm{j}$ & $838.50 \pm 57.41 \mathrm{a}-\mathrm{h}$ \\
\hline CV. \% & 9.06 & 11.22 & 4.68 & 5.39 & 6 & 6.93 & 10.72 & 11.76 & 10.94 & 5.95 \\
\hline
\end{tabular}

Means in the same column with the same letter(s) are not significantly different by Duncan's New Multiple Range Test (DMRT) at 0.05 probability level. 


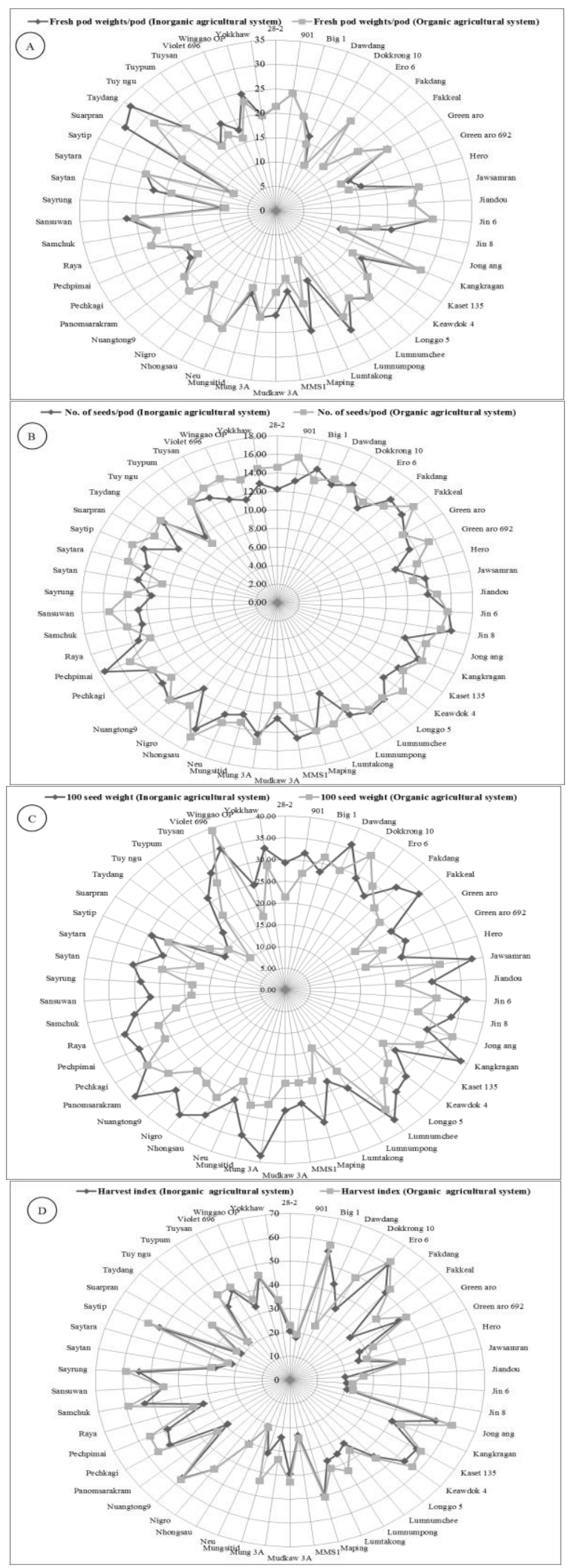

Fig 2. pod weight(A), seed numbers(B), 100 seed weight(C) and harvest index(D) of Vigna spp. 
Table 3. Means for total anthocyanin content, total phenolic content, and antioxidant capacity determined by DPPH and TEAC methods (Mean \pm SE)

\begin{tabular}{|c|c|c|c|c|c|c|c|c|}
\hline \multirow[b]{2}{*}{ Genotypes } & \multicolumn{2}{|c|}{ TAC (mg C3GE/100 g Sample) } & \multicolumn{2}{|c|}{ TPC(mg GAE/100g of Sample) } & \multicolumn{2}{|l|}{ TEAC(\%Inhibition) } & \multicolumn{2}{|l|}{ DPPH(\%Inhibition) } \\
\hline & $\begin{array}{l}\text { Inorganic } \\
\text { agricultural system }\end{array}$ & $\begin{array}{l}\text { Organic } \\
\text { agricultural system }\end{array}$ & $\begin{array}{l}\text { Inorganic } \\
\text { agricultural system }\end{array}$ & $\begin{array}{l}\text { Organic } \\
\text { agricultural system }\end{array}$ & $\begin{array}{l}\text { Inorganic } \\
\text { agricultural system }\end{array}$ & $\begin{array}{l}\text { Organic } \\
\text { agricultural system }\end{array}$ & $\begin{array}{l}\text { Inorganic } \\
\text { agricultural system }\end{array}$ & $\begin{array}{l}\text { Organic } \\
\text { agricultural system }\end{array}$ \\
\hline $28-2$ & $37.40 \pm 10.09 \mathrm{~d}$ & $89.51 \pm 17.02 \mathrm{e}-\mathrm{g}$ & $4.57 \pm 1.28 \mathrm{a}-\mathrm{f}$ & $4.67 \pm 0.23 \mathrm{a}-\mathrm{f}$ & $84.32 \pm 0.91 \mathrm{f}-\mathrm{j}$ & $85.15 \pm 1.26 \mathrm{~b}-\mathrm{k}$ & $5.90 \pm 0.88 \mathrm{~g}-\mathrm{i}$ & $8.97 \pm 1.16 \mathrm{~h}-1$ \\
\hline 901 & $85.70 \pm 6.52 \mathrm{~cd}$ & $65.64 \pm 17.24 \mathrm{e}-\mathrm{g}$ & $4.78 \pm 0.82 \mathrm{a}-\mathrm{f}$ & $4.50 \pm 0.86 \mathrm{~b}-\mathrm{g}$ & $71.69 \pm 3.01 \mathrm{no}$ & $67.93 \pm 5.58 \mathrm{q}-\mathrm{v}$ & $13.91 \pm 0.49 \mathrm{c}-\mathrm{e}$ & $16.92 \pm 3.89 \mathrm{~b}-1$ \\
\hline Big 1 & $33.70 \pm 11.40 \mathrm{~d}$ & $36.07 \pm 10.21 \mathrm{fg}$ & $3.29 \pm 0.07 \mathrm{c}-\mathrm{f}$ & $3.25 \pm 0.04 \mathrm{e}-\mathrm{h}$ & $81.48 \pm 2.07 \mathrm{~h}-\mathrm{k}$ & $83.44 \pm 1.03 \mathrm{c}-\mathrm{r}$ & $7.80 \pm 0.42 \mathrm{e}-\mathrm{i}$ & $9.03 \pm 1.85 \mathrm{~h}-1$ \\
\hline Dawdang & $388.50 \pm 12.40 \mathrm{ab}$ & $603.83 \pm 12.76 \mathrm{a}$ & $4.20 \pm 0.58 \mathrm{a}-\mathrm{f}$ & $6.18 \pm 0.40 \mathrm{ab}$ & $74.38 \pm 5.561-\mathrm{n}$ & $66.16 \pm 4.47 \mathrm{r}-\mathrm{v}$ & $18.01 \pm 1.54 \mathrm{c}$ & $23.40 \pm 5.24 \mathrm{bc}$ \\
\hline Dokkrong 10 & $57.04 \pm 0.79 \mathrm{~cd}$ & $59.43 \pm 8.34 \mathrm{fg}$ & $5.23 \pm 0.18 \mathrm{a}-\mathrm{d}$ & $5.08 \pm 0.07 \mathrm{a}-\mathrm{d}$ & $55.29 \pm 1.80 \mathrm{pq}$ & $61.92 \pm 6.92$ uv & $8.45 \pm 0.01 \mathrm{e}-\mathrm{i}$ & $18.02 \pm 4.86 \mathrm{~b}-\mathrm{h}$ \\
\hline Ero 6 & $61.80 \pm 16.70 \mathrm{~cd}$ & $68.09 \pm 17.47 \mathrm{fg}$ & $4.56 \pm 0.60 \mathrm{a}-\mathrm{f}$ & $4.57 \pm 0.61 \mathrm{~b}-\mathrm{g}$ & $87.21 \pm 2.47 \mathrm{~b}-\mathrm{h}$ & $84.23 \pm 3.79 \mathrm{c}-1$ & $10.04 \pm 0.88 \mathrm{~d}-\mathrm{i}$ & $10.35 \pm 3.39 \mathrm{e}-1$ \\
\hline Fakdang & $293.90 \pm 16.80 \mathrm{a}-\mathrm{c}$ & $262.56 \pm 18.25 \mathrm{~b}-\mathrm{f}$ & $5.11 \pm 0.87 \mathrm{a}-\mathrm{e}$ & $6.42 \pm 0.24 \mathrm{a}^{\circ}$ & $79.17 \pm 6.27 \mathrm{j}-\mathrm{m}$ & $75.31 \pm 2.821-\mathrm{q}$ & $34.20 \pm 1.33 \mathrm{a}$ & $35.61 \pm 1.46 \mathrm{a}$ \\
\hline Fakkeal & $92.21 \pm 4.25 \mathrm{~cd}$ & $235.12 \pm 14.41 \mathrm{c}-\mathrm{g}$ & $4.01 \pm 0.70 \mathrm{a}-\mathrm{f}$ & $5.66 \pm 0.14 \mathrm{a}-\mathrm{c}$ & $87.97 \pm 1.61 \mathrm{~b}-\mathrm{g}$ & $88.38 \pm 3.14 \mathrm{a}-\mathrm{f}$ & $30.08 \pm 1.77 \mathrm{ab}$ & $30.14 \pm 1.13 \mathrm{ab}$ \\
\hline Green aro & $59.30 \pm 16.09 \mathrm{~cd}$ & $64.09 \pm 10.54 \mathrm{e}-\mathrm{g}$ & $3.34 \pm 0.53 c-f$ & $2.68 \pm 0.65 \mathrm{f}-\mathrm{h}$ & $89.56 \pm 2.31 \mathrm{a}-\mathrm{f}$ & $89.44 \pm 0.97 \mathrm{a}$-e & $7.28 \pm 0.08 \mathrm{f}-\mathrm{i}$ & $8.72 \pm 1.93 \mathrm{~h}-1$ \\
\hline Green aro 692 & $55.40 \pm 3.06 \mathrm{~cd}$ & $152.29 \pm 17.35 \mathrm{c}-\mathrm{g}$ & $3.17 \pm 0.08 \mathrm{c}-\mathrm{f}$ & $3.12 \pm 0.14 \mathrm{e}-\mathrm{h}$ & $84.27 \pm 1.03 \mathrm{f}-\mathrm{j}$ & $83.02 \pm 1.03 \mathrm{c}-\mathrm{m}$ & $9.75 \pm 1.06 \mathrm{~d}-\mathrm{i}$ & $11.84 \pm 2.95 \mathrm{e}-1$ \\
\hline Hero & $104.61 \pm 16.67 \mathrm{~cd}$ & $130.90 \pm 6.67 \mathrm{~d}-\mathrm{g}$ & $5.64 \pm 0.45 \mathrm{ab}$ & $5.64 \pm 0.45 \mathrm{a}-\mathrm{c}$ & $74.22 \pm 1.081-n$ & $79.18 \pm 4.72 \mathrm{~g}-\mathrm{o}$ & $11.40 \pm 0.74 \mathrm{~d}-\mathrm{i}$ & $11.35 \pm 2.39 \mathrm{e}-1$ \\
\hline Jawsamran & $121.60 \pm 24.49 b c$ & $134.90 \pm 16.67 \mathrm{~d}-\mathrm{g}$ & $3.39 \pm 0.25 \mathrm{c}-\mathrm{f}$ & $3.65 \pm 0.10 \mathrm{~d}-\mathrm{h}$ & $84.50 \pm 1.13 \mathrm{f}-\mathrm{j}$ & $89.44 \pm 2.03 a-e$ & $11.24 \pm 0.80 \mathrm{~d}-\mathrm{i}$ & $10.76 \pm 0.43 \mathrm{f}-1$ \\
\hline Jiandou & $74.81 \pm 9.52 \mathrm{~cd}$ & $94.85 \pm 15.29 \mathrm{e}-\mathrm{g}$ & $3.16 \pm 0.01 \mathrm{c}-\mathrm{f}$ & $3.13 \pm 0.04 \mathrm{e}-\mathrm{h}$ & $82.59 \pm 3.06 \mathrm{~g}-\mathrm{k}$ & $78.01 \pm 4.76$ i-p & $10.17 \pm 1.16 \mathrm{~d}-\mathrm{i}$ & $13.53 \pm 1.33 \mathrm{~d}-\mathrm{j}$ \\
\hline $\operatorname{Jin} 6$ & $144.72 \pm 14.18 \mathrm{bc}$ & $168.32 \pm 15.62 \mathrm{c}-\mathrm{g}$ & $4.98 \pm 0.92 \mathrm{a}-\mathrm{f}$ & $4.90 \pm 0.02 \mathrm{a}-\mathrm{e}$ & $76.65 \pm 1.89 \mathrm{k}-\mathrm{n}$ & $70.42 \pm 2.85 \mathrm{o}-\mathrm{u}$ & $12.69 \pm 0.49 \mathrm{c}-\mathrm{f}$ & $14.44 \pm 1.31 \mathrm{~d}-\mathrm{j}$ \\
\hline Jin 8 & $38.80 \pm 16.28 \mathrm{~d}$ & $366.04 \pm 11.76 \mathrm{~b}-\mathrm{d}$ & $4.40 \pm 0.31 \mathrm{a}-\mathrm{f}$ & $3.81 \pm 0.54 \mathrm{c}-\mathrm{h}$ & $75.18 \pm 2.681-\mathrm{n}$ & $75.88 \pm 5.72 \mathrm{k}-\mathrm{q}$ & $15.61 \pm 0.97 \mathrm{~cd}$ & $15.34 \pm 1.18 \mathrm{~b}-\mathrm{j}$ \\
\hline Jong ang & $51.80 \pm 14.20 \mathrm{~cd}$ & $61.45 \pm 14.79 \mathrm{e}-\mathrm{g}$ & $3.22 \pm 0.36 \mathrm{c}-\mathrm{f}$ & $3.11 \pm 0.31 \mathrm{e}-\mathrm{h}$ & $73.08 \pm 5.50 \mathrm{mn}$ & $73.60 \pm 4.69 \mathrm{~m}-\mathrm{s}$ & $9.31 \pm 0.99 \mathrm{~d}-\mathrm{i}$ & $12.67 \pm 1.54 \mathrm{~d}-\mathrm{k}$ \\
\hline Kangkragan & $207.53 \pm 12.12 b c$ & $480.59 \pm 9.71 \mathrm{ab}$ & $4.15 \pm 0.93 \mathrm{a}-\mathrm{f}$ & $2.99 \pm 0.54 \mathrm{e}-\mathrm{h}$ & $85.49 \pm 7.70 \mathrm{e}-1$ & $92.38 \pm 2.85 \mathrm{a}-\mathrm{d}$ & $7.30 \pm 1.21 \mathrm{f}-\mathrm{i}$ & $8.77 \pm 1.31 \mathrm{~h}-1$ \\
\hline Kaset 135 & $116.54 \pm 12.76 \mathrm{bc}$ & $109.54 \pm 10.09 \mathrm{e}-\mathrm{g}$ & $3.46 \pm 0.78 \mathrm{c}-\mathrm{f}$ & $3.52 \pm 0.88 \mathrm{~d}-\mathrm{h}$ & $77.20 \pm 0.85 \mathrm{k}-\mathrm{n}$ & $78.54 \pm 1.74 \mathrm{~h}-\mathrm{p}$ & $8.60 \pm 0.60 \mathrm{e}-\mathrm{i}$ & $10.10 \pm 2.27 \mathrm{~h}-1$ \\
\hline Keawdok 4 & $92.20 \pm 10.33 \mathrm{~cd}$ & $92.49 \pm 7.57 \mathrm{e}-\mathrm{g}$ & $3.71 \pm 0.22 \mathrm{a}-\mathrm{f}$ & $4.09 \pm 0.51 \mathrm{c}-\mathrm{h}$ & $88.84 \pm 2.22 \mathrm{~b}-\mathrm{g}$ & $89.79 \pm 1.07 \mathrm{a}-\mathrm{e}$ & $11.91 \pm 0.06 \mathrm{c}-\mathrm{g}$ & $7.32 \pm 1.70 \mathrm{j}-1$ \\
\hline Longgo 5 & $90.90 \pm 10.08 \mathrm{~cd}$ & $91.15 \pm 4.01 \mathrm{e}-\mathrm{g}$ & $3.59 \pm 0.91 \mathrm{~b}-\mathrm{f}$ & $3.85 \pm 0.98 \mathrm{c}-\mathrm{h}$ & $90.48 \pm 2.31 \mathrm{a}-\mathrm{f}$ & $94.31 \pm 1.13 \mathrm{ab}$ & $9.49 \pm 1.34 \mathrm{~d}-\mathrm{i}$ & $11.56 \pm 2.18 \mathrm{e}-1$ \\
\hline Lumnumchee & $50.80 \pm 16.20 \mathrm{~cd}$ & $61.45 \pm 2.31 \mathrm{e}-\mathrm{g}$ & $3.55 \pm 0.93 \mathrm{~b}-\mathrm{f}$ & $3.55 \pm 0.93 \mathrm{~d}-\mathrm{h}$ & $90.07 \pm 3.35 \mathrm{a}-\mathrm{f}$ & $91.62 \pm 1.38 \mathrm{a}-\mathrm{d}$ & $9.96 \pm 1.57 \mathrm{~d}-\mathrm{i}$ & $8.70 \pm 1.62 \mathrm{~h}-1$ \\
\hline Lumnumpong & $30.80 \pm 6.14 d$ & $64.12 \pm 9.31 \mathrm{e}-\mathrm{g}$ & $2.71 \pm 0.05 \mathrm{f}$ & $2.47 \pm 0.30 \mathrm{~h}$ & $77.69 \pm 2.18 \mathrm{k}-\mathrm{n}$ & $78.14 \pm 1.26 \mathrm{~h}-\mathrm{p}$ & $7.20 \pm 0.01 \mathrm{f}-\mathrm{i}$ & $8.45 \pm 0.98 \mathrm{i}-1$ \\
\hline Lumtakong & $129.01 \pm 17.03 b c$ & $166.10 \pm 10.83 \mathrm{c}-\mathrm{g}$ & $4.82 \pm 0.39 \mathrm{a}-\mathrm{f}$ & $4.77 \pm 0.78 \mathrm{a}-\mathrm{e}$ & $589.25 \pm 7.04 \mathrm{p}$ & $64.38 \pm 5.03 \mathrm{t}-\mathrm{v}$ & $15.10 \pm 0.64 \mathrm{~cd}$ & $19.86 \pm 2.49 \mathrm{~b}-\mathrm{f}$ \\
\hline Maping & $45.92 \pm 8.96 \mathrm{~d}$ & $47.71 \pm 9.67 \mathrm{fg}$ & $2.72 \pm 0.58 \mathrm{f}$ & $2.59 \pm 0.39 \mathrm{gh}$ & $77.86 \pm 1.48 \mathrm{k}-\mathrm{n}$ & $82.31 \pm 0.89 \mathrm{~d}-\mathrm{m}$ & $6.89 \pm 0.67 \mathrm{f}-\mathrm{i}$ & $9.18 \pm 0.20 \mathrm{~h}-1$ \\
\hline MMS1 & $212.42 \pm 14.36 b c$ & $273.56 \pm 18.45 b-f$ & $2.98 \pm 0.40 \mathrm{ef}$ & $2.92 \pm 0.35 \mathrm{e}-\mathrm{h}$ & $93.23 \pm 3.02 \mathrm{ab}$ & $95.10 \pm 0.29 \mathrm{a}$ & $6.88 \pm 0.55 f-i$ & $5.96 \pm 0.40 \mathrm{j}-1$ \\
\hline mudkaw 3A & $62.81 \pm 14.49 \mathrm{~cd}$ & $125.58 \pm 9.41 \mathrm{e}-\mathrm{g}$ & $3.37 \pm 0.42 \mathrm{c}-\mathrm{f}$ & $3.76 \pm 0.76 \mathrm{c}-\mathrm{h}$ & $89.38 \pm 0.68 \mathrm{a}-\mathrm{f}$ & $91.95 \pm 0.79 \mathrm{a}-\mathrm{d}$ & $5.08 \pm 0.45 \mathrm{hi}$ & $2.98 \pm 0.101$ \\
\hline Mung 3A & $71.63 \pm 15.37 \mathrm{~cd}$ & $297.91 \pm 10.43 \mathrm{~b}-\mathrm{e}$ & $4.36 \pm 0.41 \mathrm{a}-\mathrm{f}$ & $4.88 \pm 0.05 \mathrm{a}-\mathrm{e}$ & $76.50 \pm 6.97 \mathrm{k}-\mathrm{n}$ & $84.52 \pm 1.74 c-1$ & $11.44 \pm 0.90 \mathrm{~d}-\mathrm{h}$ & $19.46 \pm 2.63 \mathrm{~b}-\mathrm{g}$ \\
\hline Mungsitid & $193.71 \pm 13.45 b c$ & $233.78 \pm 4.63 \mathrm{c}-\mathrm{g}$ & $5.26 \pm 0.55 \mathrm{a}-\mathrm{c}$ & $4.35 \pm 0.15 \mathrm{~b}-\mathrm{h}$ & $78.07 \pm 3.20 \mathrm{k}-\mathrm{n}$ & $81.44 \pm 2.89 \mathrm{e}-\mathrm{n}$ & $15.25 \pm 0.52 \mathrm{~cd}$ & $15.16 \pm 2.15 \mathrm{c}-\mathrm{j}$ \\
\hline $\mathrm{Neu}$ & $231.11 \pm 11.60 \mathrm{bc}$ & $232.12 \pm 10.41 \mathrm{c}-\mathrm{g}$ & $3.97 \pm 0.69 \mathrm{a}-\mathrm{f}$ & $3.91 \pm 0.76 \mathrm{c}-\mathrm{h}$ & $88.18 \pm 1.43 \mathrm{~b}-\mathrm{g}$ & $87.61 \pm 1.27 \mathrm{a}-\mathrm{h}$ & $9.35 \pm 0.89 \mathrm{~d}-\mathrm{i}$ & $13.16 \pm 1.88 \mathrm{~d}-\mathrm{k}$ \\
\hline Nhongsau & $79.02 \pm 11.68 \mathrm{~cd}$ & $113.55 \pm 18.99 \mathrm{e}-\mathrm{g}$ & $3.75 \pm 0.64 \mathrm{a}-\mathrm{f}$ & $3.75 \pm 0.64 \mathrm{c}-\mathrm{h}$ & $85.35 \pm 0.87 \mathrm{e}-\mathrm{j}$ & $85.46 \pm 2.06 \mathrm{~b}-\mathrm{j}$ & $6.90 \pm 0.25 \mathrm{f}-\mathrm{i}$ & $8.32 \pm 1.19 \mathrm{i}-1$ \\
\hline Nigro & $101.10 \pm 23.93 \mathrm{~cd}$ & $110.55 \pm 18.99 \mathrm{e}-\mathrm{g}$ & $3.49 \pm 0.27 \mathrm{c}-\mathrm{f}$ & $3.38 \pm 0.06 \mathrm{~d}-\mathrm{h}$ & $82.53 \pm 0.75 \mathrm{~g}-\mathrm{k}$ & $85.92 \pm 2.11 \mathrm{a}-\mathrm{j}$ & $13.87 \pm 2.24 \mathrm{c}-\mathrm{f}$ & $10.53 \pm 1.26 \mathrm{~g}-1$ \\
\hline Nuangtong9 & $60.11 \pm 10.54 \mathrm{~cd}$ & $62.59 \pm 11.79 \mathrm{e}-\mathrm{g}$ & $4.55 \pm 0.67 \mathrm{a}-\mathrm{f}$ & $2.74 \pm 0.39 \mathrm{f}-\mathrm{h}$ & $87.94 \pm 2.70 \mathrm{~b}-\mathrm{h}$ & $87.39 \pm 3.20 \mathrm{a}-\mathrm{j}$ & $9.49 \pm 0.60 \mathrm{~d}-\mathrm{i}$ & $7.28 \pm 1.85 \mathrm{j}-1$ \\
\hline Panomsarakram & $177.72 \pm 14.79 b c$ & $63.49 \pm 5.50 \mathrm{e}-\mathrm{g}$ & $3.18 \pm 0.29 \mathrm{c}-\mathrm{f}$ & $4.13 \pm 0.03 \mathrm{c}-\mathrm{h}$ & $94.94 \pm 0.38 \mathrm{a}$ & $95.36 \pm 0.29 \mathrm{a}$ & $4.86 \pm 0.65 \mathrm{i}$ & $3.88 \pm 1.30 \mathrm{kl}$ \\
\hline Pechkagi & $171.09 \pm 15.82 \mathrm{bc}$ & $440.59 \pm 19.71 \mathrm{ab}$ & $4.24 \pm 0.80 \mathrm{a}-\mathrm{f}$ & $4.08 \pm 0.65 \mathrm{c}-\mathrm{h}$ & $66.97 \pm 2.65 \mathrm{o}$ & $69.30 \pm 2.13 p-u$ & $7.94 \pm 0.23 \mathrm{e}-\mathrm{i}$ & $10.80 \pm 1.59 \mathrm{f}-1$ \\
\hline Pechpimai & $103.08 \pm 20.10 \mathrm{bc}$ & $211.07 \pm 13.10 \mathrm{c}-\mathrm{g}$ & $5.03 \pm 0.77 \mathrm{a}-\mathrm{e}$ & $4.44 \pm 0.22 \mathrm{~b}-\mathrm{h}$ & $88.20 \pm 2.23 \mathrm{~b}-\mathrm{g}$ & $78.55 \pm 2.64 \mathrm{~h}-\mathrm{p}$ & $25.15 \pm 2.76 b$ & $33.86 \pm 1.13 \mathrm{a}$ \\
\hline Raya & $44.01 \pm 14.45 \mathrm{~d}$ & $109.54 \pm 14.71 \mathrm{e}-\mathrm{g}$ & $3.09 \pm 0.55 \mathrm{~d}-\mathrm{f}$ & $3.10 \pm 0.18 \mathrm{e}-\mathrm{h}$ & $92.69 \pm 1.18 \mathrm{a}-\mathrm{c}$ & $91.11 \pm 0.85 \mathrm{a}-\mathrm{d}$ & $6.77 \pm 0.72 \mathrm{f}-\mathrm{i}$ & $6.99 \pm 1.69 \mathrm{j}-1$ \\
\hline Samchuk & $87.83 \pm 24.06 \mathrm{~cd}$ & $88.83 \pm 16.68 \mathrm{e}-\mathrm{g}$ & $3.95 \pm 0.65 \mathrm{a}-\mathrm{f}$ & $3.83 \pm 0.69 \mathrm{c}-\mathrm{h}$ & $74.52 \pm 1.501-\mathrm{n}$ & $75.11 \pm 0.121-\mathrm{r}$ & $7.22 \pm 0.29 \mathrm{f}-\mathrm{i}$ & $8.58 \pm 2.05 \mathrm{~h}-1$ \\
\hline Sansuwan & $70.12 \pm 14.82 \mathrm{~cd}$ & $80.23 \pm 14.69 \mathrm{e}-\mathrm{g}$ & $3.44 \pm 0.36 \mathrm{c}-\mathrm{f}$ & $3.55 \pm 0.49 \mathrm{~d}-\mathrm{h}$ & $91.18 \pm 1.70 \mathrm{a}-\mathrm{e}$ & $89.74 \pm 3.91 \mathrm{a}-\mathrm{e}$ & $9.34 \pm 0.61 \mathrm{~d}-\mathrm{i}$ & $9.42 \pm 3.36 \mathrm{~h}-1$ \\
\hline Sayrung & $159.40 \pm 6.96 \mathrm{bc}$ & $191.04 \pm 25.58 \mathrm{c}-\mathrm{g}$ & $4.48 \pm 0.65 \mathrm{a}-\mathrm{f}$ & $4.49 \pm 0.65 \mathrm{~b}-\mathrm{g}$ & $66.97 \pm 1.71 \mathrm{o}$ & $64.77 \pm 2.74 \mathrm{~s}-\mathrm{v}$ & $13.07 \pm 1.68 \mathrm{c}-\mathrm{f}$ & $20.54 \pm 2.29 \mathrm{~b}-\mathrm{e}$ \\
\hline Saytan & $145.93 \pm 11.46 \mathrm{bc}$ & $180.88 \pm 11.48 \mathrm{c}-\mathrm{g}$ & $4.51 \pm 0.30 \mathrm{a}-\mathrm{f}$ & $4.53 \pm 0.34 \mathrm{~b}-\mathrm{g}$ & $80.17 \pm 0.71 \mathrm{i}-\mathrm{k}$ & $80.98 \pm 0.82 \mathrm{e}-\mathrm{n}$ & $12.94 \pm 1.45 \mathrm{c}-\mathrm{f}$ & $10.07 \pm 3.60 \mathrm{~h}-1$ \\
\hline Saytara & $278.41 \pm 17.20 \mathrm{abc}$ & $252.49 \pm 19.58 \mathrm{c}-\mathrm{g}$ & $5.69 \pm 0.71 \mathrm{a}$ & $4.08 \pm 0.24 \mathrm{c}-\mathrm{h}$ & $86.48 \pm 1.35 \mathrm{c}-\mathrm{h}$ & $88.99 \pm 1.14 \mathrm{a}-\mathrm{f}$ & $12.07 \pm 4.20 \mathrm{c}-\mathrm{g}$ & $12.71 \pm 4.39 \mathrm{~d}-\mathrm{k}$ \\
\hline Saytip & $77.81 \pm 11.87 \mathrm{~cd}$ & $82.14 \pm 8.02 \mathrm{e}-\mathrm{g}$ & $4.34 \pm 0.01 \mathrm{a}-\mathrm{f}$ & $4.35 \pm 1.01 \mathrm{~b}-\mathrm{h}$ & $73.75 \pm 1.41 \mathrm{mn}$ & $77.42 \pm 1.04 j-p$ & $9.43 \pm 0.20 \mathrm{~d}-\mathrm{i}$ & $11.28 \pm 3.42 \mathrm{f}-1$ \\
\hline Suarpran & $82.22 \pm 7.31 \mathrm{~cd}$ & $83.83 \pm 14.69 \mathrm{e}-\mathrm{g}$ & $2.79 \pm 0.59 \mathrm{f}$ & $3.15 \pm 0.54 \mathrm{e}-\mathrm{h}$ & $74.97 \pm 3.561-\mathrm{n}$ & $72.27 \pm 1.14 \mathrm{n}-\mathrm{t}$ & $9.46 \pm 0.96 \mathrm{~d}-\mathrm{i}$ & $11.60 \pm 1.67 \mathrm{e}-1$ \\
\hline Taydang & $99.62 \pm 12.30 \mathrm{~cd}$ & $103.74 \pm 18.31 \mathrm{e}-\mathrm{g}$ & $3.51 \pm .07 \mathrm{~b}-\mathrm{f}$ & $3.83 \pm 0.62 \mathrm{c}-\mathrm{h}$ & $36.16 \pm 1.51 \mathrm{r}$ & $41.96 \pm 3.75 \mathrm{w}$ & $12.80 \pm 0.30 \mathrm{c}-\mathrm{f}$ & $16.77 \pm 1.84 \mathrm{~b}-\mathrm{i}$ \\
\hline Tuy ngu & $63.14 \pm 11.54 \mathrm{~cd}$ & $71.71 \pm 8.66 \mathrm{e}-\mathrm{g}$ & $3.01 \pm 0.47 \mathrm{ef}$ & $3.04 \pm 0.51 \mathrm{e}-\mathrm{h}$ & $75.07 \pm 1.161-\mathrm{n}$ & $79.56 \pm 1.23 \mathrm{f}-\mathrm{o}$ & $5.27 \pm 0.44 \mathrm{hi}$ & $6.60 \pm 1.23 \mathrm{j}-1$ \\
\hline Tuypum & $205.51 \pm 17.12 b c$ & $480.43 \pm 11.65 \mathrm{ab}$ & $4.55 \pm 0.28 \mathrm{a}-\mathrm{f}$ & $6.50 \pm 2.34 \mathrm{a}$ & $87.35 \pm 1.22 \mathrm{~b}-\mathrm{h}$ & $69.63 \pm 3.45 \mathrm{p}-\mathrm{u}$ & $11.38 \pm 1.66 \mathrm{~d}-\mathrm{i}$ & $24.19 \pm 2.70 \mathrm{~b}$ \\
\hline Tuysan & $498.32 \pm 27.22 \mathrm{a}$ & $470.15 \pm 21.15 \mathrm{ab}$ & $5.10 \pm 0.50 \mathrm{a}-\mathrm{e}$ & $3.48 \pm 0.34 \mathrm{~d}-\mathrm{h}$ & $92.19 \pm 3.29 \mathrm{a}-\mathrm{d}$ & $88.77 \pm 0.83 \mathrm{a}-\mathrm{f}$ & $10.60 \pm 1.31 \mathrm{~d}-\mathrm{i}$ & $12.91 \pm 2.39 \mathrm{~d}-\mathrm{k}$ \\
\hline
\end{tabular}




\begin{tabular}{|c|c|c|c|c|c|c|c|c|}
\hline Violet 696 & $334.43 \pm 20.20 \mathrm{a}-\mathrm{c}$ & $391.42 \pm 20.57 b c$ & $3.56 \pm 0.18 b-f$ & $3.72 \pm 0.15 \mathrm{c}-\mathrm{h}$ & $53.43 \pm 0.14 q$ & $60.45 \pm 6.37 \mathrm{v}$ & $15.59 \pm 1.31 \mathrm{~cd}$ & $21.07 \pm 4.84 \mathrm{~b}-\mathrm{d}$ \\
\hline Winggao OP & $86.84 \pm 24.71 \mathrm{~cd}$ & $362.03 \pm 13.15 b-d$ & $3.98 \pm 0.48 \mathrm{a}-\mathrm{f}$ & $3.34 \pm 0.94 \mathrm{~d}-\mathrm{h}$ & $89.66 \pm 4.60 \mathrm{a}-\mathrm{f}$ & $85.27 \pm 4.99 \mathrm{~b}-\mathrm{k}$ & $6.59 \pm 0.58 \mathrm{f}-\mathrm{i}$ & $9.39 \pm 2.30 \mathrm{~h}-1$ \\
\hline Yokkhaw & $40.07 \pm 11.40 \mathrm{~d}$ & $59.97 \pm 13.76 \mathrm{e}-\mathrm{g}$ & $3.03 \pm 0.14 \mathrm{ef}$ & $4.39 \pm 0.43 \mathrm{~b}-\mathrm{h}$ & $86.05 \pm 2.57 \mathrm{~d}-\mathrm{i}$ & $89.83 \pm 3.97 \mathrm{a}-\mathrm{e}$ & $8.23 \pm 0.05 \mathrm{e}-\mathrm{i}$ & $10.53 \pm 2.81 \mathrm{~g}-1$ \\
\hline CV.\% & 7.34 & 8.16 & 6.31 & 11.63 & 4.07 & 5.98 & 8.58 & 8.22 \\
\hline
\end{tabular}

Means in the same column with the same letter(s) are not significantly different by Duncan's New Multiple Range Test (DMRT) at 0.05 probability level.

Table 4. Mean squares from combined analysis of variance for system, genotypes, and interaction for two production systems.

\begin{tabular}{|c|c|c|c|c|c|c|c|}
\hline Sources of variance & $\mathrm{DF}$ & Pod length $(\mathrm{cm})$ & $\begin{array}{l}\text { No. of seeds.pod }{ }^{-1} \\
\text { (seed) }\end{array}$ & $\begin{array}{l}\text { No. of pods.plant } \\
\text { (pod) }\end{array}$ & $\begin{array}{c}\text { Fresh pod yield.plant }^{-1} \\
(\mathrm{~g})\end{array}$ & $\begin{array}{c}100 \text { seed } \\
\text { weight }(\mathrm{g})\end{array}$ & Harvest index \\
\hline Systems(S) & 1 & $4,313.86^{* *}$ & $16.85^{* *}$ & $2,079.12^{*}$ & $2,084,964.17^{*}$ & $724.04^{*}$ & $63.28 *$ \\
\hline Blocks. Within S & 6 & $1,227.78 \mathrm{~ns}$ & $14.44 \mathrm{~ns}$ & $1,002.28 \mathrm{~ns}$ & $71,722.12 \mathrm{~ns}$ & $316.24 \mathrm{~ns}$ & $38.19 \mathrm{~ns}$ \\
\hline Genotypes (G) & 49 & $4,749.10 * *$ & $56.22 * *$ & $2,629.00 * *$ & $4,749,423.99 * *$ & $3,999.61 * *$ & $111.86^{* *}$ \\
\hline$S \times G$ & 49 & $4,486.57 * *$ & $49.25 * *$ & $2,146.96 * *$ & $4,465,499.02 * *$ & $2,230.15 * *$ & $78.69 * *$ \\
\hline Pooled error & 294 & 604.65 & 7.68 & 489.20 & 430332.68 & 180.11 & 15.98 \\
\hline
\end{tabular}

Table 5. Means for pod lenght, pod weight, seeds/plant, pods/plant 100 seed weight and harvest index of Vigna spp. at two growing systems.

\begin{tabular}{|c|c|c|c|c|c|c|c|}
\hline Systems & $\begin{array}{l}\text { Pod length } \\
\text { (cm) }\end{array}$ & $\begin{array}{c}\text { Fresh pod } \\
\text { weight.pod }^{-1}(\mathrm{~g})\end{array}$ & $\begin{array}{l}\text { No. of seeds.pod }{ }^{-1} \\
\text { (seed) }\end{array}$ & $\begin{array}{l}\text { No. of pods.plant }{ }^{-1} \\
\text { (pod) }\end{array}$ & $\begin{array}{c}\text { Fresh pod } \\
\text { yield.plant }^{-1}(\mathrm{~g})\end{array}$ & $\begin{array}{c}100 \text { seed } \\
\text { weight }(\mathrm{g})\end{array}$ & $\begin{array}{l}\text { Harves } \\
\text { index }\end{array}$ \\
\hline Inorganic & $45.25 \mathrm{a}$ & $21.60 \mathrm{a}$ & $13.96 \mathrm{a}$ & $41.34 \mathrm{a}$ & $849.19 \mathrm{a}$ & $29.86 \mathrm{a}$ & $37.44 \mathrm{~b}$ \\
\hline Organic & $46.57 \mathrm{a}$ & $20.82 \mathrm{~b}$ & $13.38 \mathrm{a}$ & $34.78 \mathrm{~b}$ & $718.99 \mathrm{~b}$ & $24.89 \mathrm{~b}$ & $40.53 \mathrm{a}$ \\
\hline Means & 45.91 & 21.21 & 13.67 & 38.06 & 784.09 & 27.38 & 38.99 \\
\hline
\end{tabular}

Means in the same column with the same letter(s) are not significantly different by Duncan's New Multiple Range Test (DMRT) at 0.05 probability level. 
Chavas et al., 2009). Vigna spp. genotypes have high variations in pod color, starch properties and nutritionally important components (Peyrano et al, 2016). The pod colors of Vigna spp. include white, green yellow, green, purple, brown, red and black. Previous study indicated that pod colors of Vigna spp. were closely related to phytochemical constituents and concentrations. In this study, Vigna spp. genotypes with white, green, purple, brown, red and black colors were selected because they are rich in anthocyanins and phenolic compounds, and the results indicated that Dawdang (organic agricultural system) had the highest total anthocyanin content at edible stage $(603.83 \pm 12.76 \mathrm{mg}$ CGE. $100 \mathrm{~g}^{-1}$ of fresh weight (FW). In previous investigations with many crops, total anthocyanin contents were $0.59 \pm 0.01$ mg CGE. $100 \mathrm{~g}^{-1}$ in brown colored common bean (Dzomba et al. 2013). Total anthocyanin contents (ranging from $30.80 \pm 6.14$ to $603.83 \pm 12.76 \mathrm{mg} \mathrm{CGE} .100 \mathrm{~g}^{-1}$ of $\mathrm{FW}$ in fresh pod) in Vigna spp. in this study were higher than those in previous study. The differences in the results among different studies were due largely to plant species and plant genotypes and plant parts. The results in this study and previous studies indicate that there is variation in anthocyanin content in Vigna spp. and breeding for high anthocyanins in Vigna spp. is possible. Moreover, the results showed that many genotypes of bean planted under organic agricultural system had height antioxidants and their capacities than inorganic agricultural system. The significant interaction between system and genotype effects indicated that genotype responded differently to changes in system production. High proportion of variation on yield and yield components were found for the genotype effect, therefore more testing sites are needed or the environments in locations need to be controlled (Gurung et al., 2012; El-Shaieny et al., 2015). Many study researchers and many plants reported that yield and yield components content are affected by genetic and environment conditions. Moreover, Singh et al. (2006) and Adeyanju et al. (2012) reported environment has stronger effect on yield. In contrast, Gurung et al. (2012) found that genotype plays a major role in yield contents as more than $70 \%$ of the variation was due to cultivar effect although $\mathrm{SxG}$ were significant. However, most genotypes were local varieties in countries. Hence, this experiment is to study in real conditions and Kangkragan genotype should be used for further breeding or plant in commercial genotypes. Because this genotype can adapt and give height yields under two production systems.

\section{Materials and Methods}

\section{Plant materials}

Fifty genotypes of yardlong bean and cowpea including 28-2, 901, Big 1, Jin 6, Jin 8, Dawdang, Dokkrong 10, Ero 6, Fakdang, Fakkeal, Green aro, Green aro 692, Hero, Jawsamran, Jiandou, Jong ang, Kangkragan, Kaset 135, keawdok 4, Longgo 5, Lumnumchee, Lumnumpong, Lumtakong, Maping, MMS1, mudkaw 3A, Mung 3A, Mungsitid, Neu, Nhongsau, Nigro, Nuangtong 9, Panomsarakram, Pechkagi, pechpimai, Raya, Samchuk, Sansuwan, Sayrung, Saytan, Saytara, Saytip, Suarpran, Taydang, Tuy ngu, Tuypum, Tuysan, Violet 696, Winggao $\mathrm{OP}$, and Yokkhaw were selected from anyplace in Thailand and other countries.

\section{Treatments and experimental design}

Fifty genotypes of Vigna spp. (treatments) were selected from someplace in many areas. Plot experiments were planted at the Department of Plant Science, Faculty of Technology and Community Development Thaksin University Phatthalung Campus, Phatthalung Thailand ( $\left.7^{\circ} 37^{\prime} 0^{\prime \prime} \mathrm{N}, 100^{\circ} 5^{\prime} 0^{\prime \prime} \mathrm{E}\right)$. The soil type was Phatthalung series (Yt: Fine, kaolinitic, isohyperthermic, plinthic, paleaquults). All genotypes of yardlong bean and cowpea were sown under field trial conditions. Before planting, sunhemps (Crotalaria juncea L.) were planted and practiced out by thoroughly ploughing for soil improvement for one season. The plot was sized 1 x 5 $\mathrm{m}\left(5 \mathrm{~m}^{2}\right)$. Seeds were dropped in each hole. Plant to plant and row to row spacing was maintained at $0.50 \mathrm{~m}$ and $0.75 \mathrm{~m}$, respectively. The rows were planted in pairs. Seven days after planting, they were separated and only one plant was left in the hole. This experiment was conducted between January and June 2015. The study was performed as a Randomized Complete Block Design (RCBD) with four replications and twenty plants.replication ${ }^{-1}$. Soil preparation and crop management for organic trail followed the International Federation of Organic Agriculture Movements (IFOAM, 2005). Inorganic fertilizer (formula 15-15-15 of NPK) was applied to the inorganic trial at the rate of $650 \mathrm{~kg}^{-h^{-1}}$ and compost manure was applied to organic trial at the same rate of $650 \mathrm{~kg} \cdot \mathrm{ha}^{-1}$. Two types of fertilizers (inorganic and organic) were applied in two splits. At first split, the fertilizers $\left(325 \mathrm{~kg}^{-h^{-1}}\right)$ were applied at the bottoms of the hills shortly before transplanting. At second split, the fertilizers $\left(325 \mathrm{~kg} \cdot \mathrm{ha}^{-1}\right)$ were applied around the stems of the plants and hilled up by hoes (pilling soil up around the base of the plant). Manual weeding was practiced for both inorganic and organic systems, and inorganic control of insects and diseases was practiced under inorganic system only (Benchasri, 2015), whereas biological control was practiced under organic farming systems (Oyesola and Obabire, 2011). Yardlong bean and cowpea harvesting was carried out when the pods were still fresh. Fresh pods were collected $5-7$ days after flowering.

\section{Data collection}

\section{Soil and meteorological condition.}

Soil contents and chemical properties were measured. Weather condition about rainfall, humidity and air temperature such as maximum, minimum and average air for this experiment were also measured monthly from plantation to harvest by a weather station located $100 \mathrm{~m}$ away from the experimental field.

\section{Yield and yield component traits}

Important morphological characteristics of Vigna spp. such as pod length, fresh pod weight, number of seeds.pod ${ }^{-1}$, number of pods.plant ${ }^{-1}$ and fresh pod yield.plant ${ }^{-1}$ were measured for 60 pods.treatment ${ }^{-1}$. In addition, 100 seed weight and harvest index were also recorded all genotypes. The pods of this crop were harvested for yield assessment at $5-7$ days after flowering.

\section{Sample Extraction and Determination of Total Anthocyanin Content (TAC)}

Anthocyanins in distinct pod parts were extracted according to the method previously described by Yang et al. (2009) and Simla et al. (2016) with slight modification. Portion of $0.5 \mathrm{~g}$ samples were put into a conical flask containing $25 \mathrm{~mL}$ of acidified methanol (methanol-1\% citric acid, 80:20 $\mathrm{v} \cdot \mathrm{v}^{-1}$ ) mixed well and stored for $24 \mathrm{~h}$ at $4^{\circ} \mathrm{C}$. The solution was then 
transferred to a tube and centrifuged at $11,538 \mathrm{xg}$ for $10 \mathrm{~min}$ at $4^{\circ} \mathrm{C}$. Further, the supernatants were collected and kept at $20^{\circ} \mathrm{C}$ in the dark until analysis.

Total anthocyanin content was measured using the $\mathrm{pH}$ differential method as described by Dzomba et al. (2013). A UV-vis spectrophotometer (UV-1700 Pharmaspec, Shimadzu, Japan) was used to measure the absorbance at 510 and $700 \mathrm{~nm}$. Anthocyanin levels were expressed as $\mathrm{mg}$ of cyanidin-3-glucoside equivalents. $\mathrm{g}^{-1}$ of fresh weight $(\mathrm{mg}$ CGE. $100 \mathrm{~g}^{-1}$ of $\mathrm{FW}$ ), using the reported molar extinction coefficient of $26900 \mathrm{M}^{-1} . \mathrm{cm}^{-1}$ and a molecular weight of 449.2 g. $\mathrm{mol}^{-1}$.

\section{Determination of Total Phenolic Content (TPC)}

Total phenolic contents were determined using FolinCiocalteu (F-C) method described by $\mathrm{Hu}$ and $\mathrm{Xu}$ (2011). Briefly, the appropriate dilutions of extracts were oxidized with F-C reagent, and the reaction was neutralized with sodium carbonate. The absorbance of the resulting blue color was measured at $765 \mathrm{~nm}$ after $90 \mathrm{~min}$, and the phenolic content was expressed as $\mathrm{mg}$ of gallic acid equivalents (GAE). $100 \mathrm{~g}^{-1}$ of fresh weight (mg GAE. $100 \mathrm{~g}^{-1}$ of $\mathrm{FW}$ ).

\section{Determination of antioxidant activity}

The capacity for scavenging DPPH radicals was assessed by measuring the bleaching of a black-coloured methanol solution containing DPPH radicals as described by Yang and Zhai (2010). Briefly, $0.1 \mathrm{mM}$ solution of methanolic DPPH solution was prepared. The initial absorbance of the DPPH in methanolic was determined at $517 \mathrm{~nm}$ and did not change throughout the period of assay. An aliquot $(0.1 \mathrm{~mL})$ of each sample (with appropriate dilution if necessary) was added with $3 \mathrm{~mL}$ of methanolic DPPH solution. Discolorations were measured at $517 \mathrm{~nm}$ after incubation for $30 \mathrm{~min}$ at $30^{\circ} \mathrm{C}$ in the dark. Measurements were performed at least in triplicate. The percentage of DPPH was calculated as:

$\% \mathrm{DPPH}$ reduction $=\left(\mathrm{Abs}_{\text {control }}-\mathrm{Abs}_{\text {sample }}\right) \times 100 / \mathrm{Abs}_{\text {control }}$ ,where $\mathrm{Abs}_{\text {control }}$ is the absorbance of the control, and $\mathrm{Abs}_{\text {sample }}$ is the absorbance of the sample.

Trolox equivalent antioxidant capacity (TEAC) assay, which measures the reduction of radical cations of ABTS by antioxidants was conducted as described by Yang and Zhai (2010). The ABTS scavenging rate was calculated as:

$\% \mathrm{ABTS}$ reduction $=\left(\mathrm{Absinitial}-\mathrm{Abs}_{\text {final }}\right) \times 100 / \mathrm{Abs}_{\text {initial }}$, where $\mathrm{Abs}_{\text {initial }}$ is the absorbance of the control, and $\mathrm{Abs}_{\text {final }}$ is the absorbance of the sample.

\section{Statistical analysis}

Data for separate locations were analyzed statistically according to a Randomized Complete Block Design. All analyses were done using the statistical programme of SPSS (Statistical Package for the Social Science for Windows) version 16.0. Significant treatment differences were separated using the Duncan's New Multiple Range Test (DMRT) at 0.05 probability level. Yield, yield components, anthocyanin content, total phenolic content and antioxidant capacity traits were statistically analyzed for each system. Error variances were tested for homogeneity with Bartlett's test as described by Gomez and Gomez (1984). Combined analysis of variance was done for two environments (production systems) according to a statistical model explained by Freeman (1973).

\section{Conclusion}

Vigna spp. plants grown in soil treated with chemical fertilizer were shown a vigorous vegetative growth (pod length, number of pods.plant ${ }^{-1}$ and fresh pod yield) compare to application of an organic agricultural system. However, organic Vigna spp. products are expected to be healthy for humans and may be more profitable than those from produced by a conventional production system. The outcome of the study may be used as guidance for Vigna spp. production in Thailand.

\section{Acknowledgments}

The authors would like to thank the Graduate School, Thaksin University and the authors also would like to thank the Research and Development Institute, Thaksin University and National Research Council of Thailand (NRCT) for funding this research.

\section{References}

Abbas G, Abbas Z, Aslam M, Malik AU, Ishaque M, Hussain F (2011) Effects of organic and inorganic fertilizers on mungbean (Vigna radiata (1.)) yield under arid climate. Int Res J Plant Sci. 2(4): 94 - 98.

Adeoye PA, Adebayo SE, Musa JJ (2011) Growth and yield response of cowpea (Vigna unguiculata) to poultry and cattle manure as amendments on sandy loam soil plot. Agri Sci. 6(5): $218-221$

Adeyanju AO, Ishiyaku MF, Echekwu CA, Olarewaju JD (2012) Generation mean analysis of dual purpose traits in cowpea (Vigna unguiculata [L.] walp). Afr J Biptechnol. 11(46): 10473 - 10483

Agyeman K, Berchie JN, Osei-Bonsu I, Tetteh Nartey E, Fordjour JK (2014) growth and yield performance of improved cowpea (Vigna unguiculata L.) varieties in Ghana Agri Sci. 2(4): $44-52$.

Aikins SHM, Afuakwa JJ (2008) Growth and dry matter yield responses of cowpea to different sowing depths. ARPN J Agric Biol Sci. 3(5 - 6): 50 - 54 .

Attigah AS, Asiedu EK, Agyarko K, Dapaah HK (2013) Growth and yield of okra (Abelmoschus esculentus L.) as affected by organic and inorganic fertilizers. ARPN J Agric Biol Sci. 8(12): 766 - 770.

Badiane FA, Gowda BS, Cisse N, Diouf D, Sadio O, Timko MP (2012) Genetic relationship on SSR markers. Genet Mol Res. 11(1): 292 - 304

Bastos EA, Folegatti MV, Faria RT, Júnior ASA, Cardoso MJ (2002) Simulation of growth and development of irrigated cowpea in Piauí State by CROPGRO model. Pesq Agropec Bras Brasília. 37(10): 1381 - 1387.

Benchasri S (2015) Effects of chemical and organic agricultural systems for okra (Abelmoschus esculentus L. Moench) production in Thailand. Aust J Crop Sci. 9(10): $968-975$.

Benchasri S, Bairaman C (2010) Evaluation of yield, yield components and consumers' satisfaction towards yardlong bean and cowpea in agricultural organic system. Bulg $\mathbf{J}$ Agric Sci. 16(6): 705 - 712

Benchasri S, Bairaman B, Nualsri C (2012) Evaluation of yardlong bean and cowpea for resistance to Aphis craccivora Koch in southern part of Thailand. J Anim Plant Sci. 22(4): $1024-1029$.

Chavas JP, Posner JL, Hedtcke JL (2009) Organic and conventional production systems in the Wisconsin 
integrated cropping systems trial: II. economic and risk analysis 1993-2006. Agron J. 101(2): 288 - 295.

Chinma CE, Emelife, IG, Alemede IC (2008) Physicochemical and functional properties of some Nigerian cowpea varieties. Pak J Nutr. 7(1): 186 - 190.

Connell SO, Shi W, Grossman JM, Hoyt GD, Fager KL, Creamer NG (2015) Short-term nitrogen mineralization from warm-season cover crops in organic farming systems. Plant Soil. 396(1): 353-367.

Drinkwater LE (2002) Cropping systems research: reconsidering agricultural experimental approaches. Horttechnology. 12(3): $355-361$.

Dzomba P, Togarepi E, Mupa M (2013) Anthocyanin content and antioxidant activities of common bean species (Phaseolus vulgaris L.) Growth in Mashonaland Central, Zimbabwe. Afr J Agric Res. 8(25): 3330 - 3333.

El-Shaieny AAH, Abdel-Ati YY, El-Damarany AM, Rashwan AM (2015) Stability analysis of components characters in cowpea (Vigna unguiculata (L.) Walp). J Hortic For. 7(2): 24 - 35.

Fang J, Roberts PA, Ehlers JD, Chih-Cheng T (2007) Genetic diversity of cowpea (Vigna unguiculata (L.) Walp) in four West African and USA breeding programs as determined by AFLP analysis. Genet Resource Crop Evol. 54(6): 1197 $-1209$.

Farahvash F, Mirshekari B (2011) Yield and yield components of cowpea as affected by different sources and application rates of nitrogen fertilizers. J Food Agric Environ. 9(3-4): 295 - 298.

Freeman GH (1973) Statistical methods for the analysis of genotype environments. Heredity. 31: 339-354.

Gomez KA and Gomez AA (1984) Statistical Procedure for Agricultural Research. John Wiley and Sons. New York.

Gurung T, Techawongstien S, Suriharn B, Techwongstien S (2012) Stability analysis of yield and capsaicinoids content in chili (Capsicum spp.) grown across six environments. Euphytica. 187(1):11 - 18 .

Hamid S, Muzaffar S, Wani IA, Masoodi FA, Bhat MM (2016) Physical and cooking characteristics of two cowpea cultivars grown in temperate Indian climate. J Saud Soc Agric Sci. 15(2): 127-134.

Henry AE, Chinedu PP (2014) Cowpea (Vigna unguiculata L. Walp) response to phosphorus fertilizer under two tillage and mulch treatments. Soil Till Res. 136: 70-75

Hu QP, Xu JG (2011) Profiles of carotenoids, anthocyanins, phenolics, and antioxidant activity of selected color waxy corn grains during maturation. J Agri Food Chem. 59: 2026 $-2033$.

Huaqiang T, Manman T, Qian L, Yongpeng Z, Jia L, Huanxiu L (2012) A review of molecular makers applied in cowpea (Vigna unguiculata L. Walp.) J Life Sci. 6: 1190 1199.

International Federation Organic Movement (2009) Die Deutsche Bibliothek. Bonn.

Karikari B, Arkorful E, Addy S (2015) Growth, nodulation and yield response of cowpea to phosphorus fertilizer application in Ghana. J Agron. 14(4): 234 - 240.

Kongjaimun A, Kaga A, Tomooka N, Somta P, Vaughan DA, Srinives P (2012) The genetics of domestication of yardlong bean, Vigna unguiculata (L.) Walp. ssp. unguiculata cv.-gr. Sesquipedalis. Ann Bot. 109: 11851200.

Lotter DW (2003) Organic agriculture. J Sustain Agric. 21(4): $59-128$.
Madukwe DK, Christo IEC, Onuh MO (2008) Effects of organic manure and cowpea (Vigna unguiculata (L.) WALP) varieties on the chemical properties of the soil and root nodulation. Sci World J. 3(1): 43 - 46.

Magani IE, Kuchinda C (2009) Effect of phosphorus fertilizer on growth, yield and crude protein content of cowpea (Vigna unguiculata [L.] Walp) in Nigeria. J Applied Bio. Sci. 23: $1387-1393$.

Mahoney PR, Olson KD, Porter PM, Huggins DR, Perillo CA, Crookston RK (2004) Profitability of organic cropping systems in Southwestern Minnesota. Renew Agr Food Syst. 19(1): $35-46$

Masvodza DR, Mazhude N, Mugabe J, Musango R (2014) Preliminary genetic diversity analysis of introduced and local Zimbabwe cowpea landraces. Afr J Agric Res. 9(49): $3571-3580$.

Mukhtar FB, Singh BB (2006) Influence of photoperiod and gibberellic Acid (GA3) on the growth and flowering of cowpea [Vigna unguiculata (L.) Walp]. Food Agric Environ. 4(2): $201-203$.

Naeem M, Iqbal J, Bakhs MAAHA (2006) Comparative study of inorganic fertilizers and organic manures on yield and yield components of mungbean (Vigna radiat L.). J Agric Soc Sci. 2(4): 227-229.

Nirmal DE, Kumar RAJ, Kalloo G (2001) Diet versatility in cowpea (Vigna unguiculata) genotypes. Indian J Agric Sci. 71(9): $598-601$

Nkaa FA, Nwokeocha OW, Ihuoma O (2014) Effect of phosphorus fertilizer on growth and yield of cowpea (Vigna unguiculata). IOSR J Pharm Biol Sci. 9(5): 74 - 82.

Ntare BR (1991) Variation in reproductive efficiency and yield of cowpea under high temperature conditions in a Sahelian environment. Euphytica. 59(1): 27 - 32 .

Odedeji JO, Oyeleke WA (2011) Comparative Studies on Functional Properties of Whole and Dehulled Cowpea Seed Flour (Vigna unguiculata). Pak J Nutr. 10(9): 899-902.

Olusegun OO (2014) Influence of NPK 15-15-15 Fertilizer and pig manure on nutrient dynamics and production of cowpea, Vigna unguiculata L. Walp. Am J Agr Forestry. 2(6): $267-273$

Oyesola OB, Obabire I (2011) Farmers' perceptions of organic farming in selected local government areas of Ekiti State, Nigeria. J Org Syst. 6(1): $20-26$.

Petlamul W, Ngampongsai A, Petcharat J (2009) Oviposition preference of papaya fruit fly, Bactrocera papayae Drew $\&$ Hancock (Diptera: Tephritidae) on some chili varieties. Thaksin J. 12(2): 43 - 49.

Peyrano F, Speroni F, Avanza MV (2016) Physicochemical and functional properties of cowpea protein isolates treated with temperature or high hydrostatic pressure. Innov Food Sci Emerg Tech. 33: 38-46.

Phansak P, Taylor PWJ, Mongkolporn O (2005) Genetic diversity in yardlong bean (Vigna unguiculata ssp. sesquipedalis) and related Vigna species using sequence tagged microsatellite site analysis. Sci Hortic. 106(2): $137-$ 146.

Reganold JP, Wachter JM (2016) Organic agriculture in the twenty-first century. Nature Plants 2(2):15221.

Sarutayophat T, Nualsri C, Santipracha Q, Saereeprasert V (2007) Characterization and genetic relatedness among 37 yardlong bean and cowpea accessions based on morphological characters and RAPD analysis. Songklanakarin J Sci Technol. 29(3): 591 - 600. 
Shevkani K, Kaur A, Kumar S, Singh N (2015) Cowpea protein isolates: functional properties and application in gluten-free rice muffins. LWT - Food Sci Technol. 63(2): $927-933$

Simla S, Boontang S, Harakotr B (2016) Anthocyanin content, total phenolic content, and antiradical capacity in different ear components of purple waxy corn at two maturation stages. Aust J Crop Sci. 10(5): 675 - 682 .

Singh BB, Ajeigbe HA, Tarawali SA, Fernandez-Rivera S, Abubakar M (2003) Improving the production and utilization of cowpea as food and fodder. Field Crops Res. 84(1-2): 169-177.

Singh I, Badaya SN, Tikka SBS (2006) combining ability for yield over environments in cowpea (Vigna unguiculata $\mathrm{L}$. Walp). Indian J Crop Sci. 1(1 - 2): 205 - 206.

Win KT, Oo AZ (2015) Genotypic difference in salinity tolerance during early vegetative growth of cowpea (Vigna unguiculata L. Walp.) from Myanmar. Biocatalysis Agric Biotechnol. 4(4): 449 - 455.
Win KT, Oo AZ, Aung HP, Terasaki A, Yokoyama T, Bellingrath-Kimura SD (2015) Varietal differences in growth and Cs allocation of blackgram (Vigna mungo) under water stress. Environ Exp Bot. 109: 244-253.

Yang Z, Chen Z, Yuan S, Zhai W, Piao X, Piao X (2009) Extraction and identification anthocyanin from purple Corn (Zea mays L.). Int Food Sci Tech. 44(12):2485 - 2492.

Yang Z, Zhai W (2010) Identification and antioxidant activity of anthocyanins extracted from the seed and cob of purple corn (Zea mays L.). Innov. Food Sci Emerg Tech. 11(1):169-176. 